\title{
GLOBAL NUMERICAL CONSTRAINTS ON TREES
}

\author{
EVERARDO BÁRCENAS ${ }^{a}$ AND JESÚS LAVALLE $^{b}$ \\ ${ }^{a}$ Universidad Politécnica de Puebla, México \\ e-mail address: ismael.barcenas@uppuebla.edu.mx \\ ${ }^{b}$ Benemérita Universidad Autónoma de Puebla, México \\ e-mail address: jlavalle@cs.buap.mx
}

\begin{abstract}
We introduce a logical foundation to reason on tree structures with constraints on the number of node occurrences. Related formalisms are limited to express occurrence constraints on particular tree regions, as for instance the children of a given node. By contrast, the logic introduced in the present work can concisely express numerical bounds on any region, descendants or ancestors for instance. We prove that the logic is decidable in single exponential time even if the numerical constraints are in binary form.

We also illustrate the usage of the logic in the description of numerical constraints on multi-directional path queries on XML documents. Furthermore, numerical restrictions on regular languages (XML schemas) can also be concisely described by the logic. This implies a characterization of decidable counting extensions of XPath queries and XML schemas. Moreover, as the logic is closed under negation, it can thus be used as an optimal reasoning framework for testing emptiness, containment and equivalence.
\end{abstract}

\section{INTRODUCTION}

XML is nowadays recognized as the standard technology in the description and exchange of data in the World Wide Web. One of the cornerstones in the XML community is XPath, which has been well-established as the most accepted query language for XML documents (finite unranked trees). XPath takes also an important role in other XML technologies, such as XSLT, XProc and XQuery. The navigational core of XPath is formed by regular path queries, and its expressive power corresponds to the first order logic with two variables $\mathrm{FO}^{2}$ Mar05]. A regular path query selects the nodes obtained by the navigation of the path. Consider for instance the following query: $\uparrow^{\star}: a / \downarrow: b$. This query expression navigates through the ancestors nodes $\left(\uparrow^{\star}\right)$ named $a$, and from there it selects the children $(\downarrow)$ labeled with $b$. The XPath language specification [CD99] also defines arithmetical constructs on the number of node occurrences, for example: $\downarrow: c\left[\downarrow^{\star}: a>\uparrow^{\star}: b\right]$. This query selects the $c$ children with more descendants named $a$ than ancestors named $b$. However, extending regular path queries with arithmetical constructs leads to undecidability tCM09. Here we

2012 ACM CCS: [Theory of Computation]: Logic-Modal and temporal logics; Logic-Automated reasoning; Formal languages and automata theory - Tree languages; [Information systems]: Data management systems - Query languages - XML query languages - XPath.

Key words and phrases: counting constraints, satisfiability, query reasoning, XML schemas.

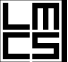

MELTHODS IN COMPUTER SCIENCE
DOI:10.2168/LMCS-10(2:10)2014

(C) E. Bárcenas and J. Lavalle (c) Creative Commons 
focus our study on numerical constraints, that is, restrictions with respect to constants (in binary), as for instance: $\downarrow: c\left[\downarrow^{\star}: a>5\right]$. In this query, the selection is constrained to the $c$ children with more than 5 descendants named $a$. In this paper, we identify decidable extensions of XPath with numerical constraints on any regular path.

Query reasoning in the presence of XML schemas is one of the central issues that arises from the static analysis of XML specifications and transformations. XML schemas are used to describe sets of trees by means of regular expressions. Regular tree languages (types) subsume most XML schema languages used in practice, such as XML schema, DTDs and RelaxNG [MLMK05]. Numerical constraints on regular languages are widely used in many technologies, such as egrep [Hum88], Perl [WCO00] and XML schema languages [MLMK05]. These constraints serve to bound the number of occurrences. For instance, the regular language over $\{a, b\}$, such that $a$ occurs exactly once and $b$ occurs at least four times, can be written as follows:

$$
\left(a b b b b^{+}\right)\left|\left(b a b b b^{+}\right)\right|\left(b b a b b^{+}\right)\left|\left(b b b a b^{+}\right)\right|\left(b b b b^{+} a\right)
$$

However, in general, hardcoding numerical constraints produces exponentially larger expressions than the original problem Gel10. This implies a drastic impact in the computational cost of reasoning on these kind of constraints, more precisely, reasoning on hardcoded numerical constraints is exponentially more expensive. Furthermore, Gelade Gel10] also showed that even if the numerical constraints are directly translated to NFAs, the exponential blowup cannot be avoided. In this paper, we provide a way to avoid this exponential blow-up by a succinct characterization of regular languages with numerical constraints. More precisely, in the current work it is proposed a tree logic with counting constructs. These constructs can restrict the number of node occurrences with respect to a constant coded in binary. It is also shown that the proposed logic is decidable in exponential time. Also, we show that regular tree expressions (and queries) with counting operators can be linearly embedded by the proposed logic.

Motivations and Related Work. The fully enriched $\mu$-calculus is the modal logic with inverse and graded modalities, nominals, a least and a greatest fixed-points. Graded modalities are used to constrain the number of immediate successors of certain node with respect to a constant. The fully enriched $\mu$-calculus was shown to be undecidable by Bonatti et al. BLMV06. Nevertheless, it has been recently shown that this result does not apply in the context of finite trees; more precisely, it was provided in BGLS11] a single exponential satisfiability algorithm for the fully enriched $\mu$-calculus for trees. However, graded modalities (in trees) are limited to impose numerical bounds on the number of children nodes only. Although, it was shown in BMM10 that numerical constraints on descendant nodes can be expressed by graded $\mu$-calculus formulas, this comes at an exponential cost in the formula size. This implies that, even at the logical level, hardcoding in-depth numerical constraints produces an exponential blow-up. In contrast, we show in this paper, that our logic can express descendant constraints without an extra cost with respect to the $\mu$-calculus. In addition, backward constraints, such as on ancestor nodes, can also be expressed for free.

Seidl et al. [SSM03] showed that the extension of monadic second order logic (MSOL) with Presburger arithmetic is undecidable. In other works [DL10, DZLM04, SSMH04, decidable extensions of tree logics with Presburger arithmetical constraints on children are broadly studied. Demri and Lugiez [DL10] provide a PSPACE bound on the decidability of modal logic extended with Presburger constraints on children nodes. When proving 
decidability of a fragment of ambient logic, Dal-Zilio et al. [DZLM04 introduced a modal tree logic with Presburger arithmetic and regular constraints. In an independent work, Seidl et al. [SSMH04 introduced a decidable extension to the logic of Dal-Zilio et al. DZLM04]. The extension consists of a fixed-point operator.

In this paper we choose a different trade-off, we propose a tree logic with less general cardinality constraints (with respect to binary constants) on more extensive tree regions (descendants, ancestors, etc.). In the same vein, it has been recently proposed Bianco et al. BMM09 a graded version of the computation tree logic CTL . This logic can pose constraints on the number of paths expressed by CTL formulas. Constraints are made with respect to constants written in unary form. In [BMM10], the same result was later extended with constants coded in binary. This approach however does not support backward navigation, neither in the graded formulas, nor in the non-graded ones. One consequence is that cardinality constraints can only be expressed on downward tree regions, as children or descendants of a given node. It should also be recalled that CTL is not as expressive as MSOL. This implies that some regular properties, as the ones in XML schemas, cannot be expressed by CTL formulas. Besides expressing numerical constraints on any multidirectional regular path, our logic is as expressive as MSOL and can concisely capture regular tree languages (XML schemas).

The notion of global constraints has been also subject of recent study in $\left[\mathrm{BCG}^{+} 10\right.$, $\mathrm{BCG}^{+} 13$. Burgoño et al. $\mathrm{BCG}^{+} 10, \mathrm{BCG}^{+} 13$ introduced an automata model capable to test (dis)equality modulo a given flat equational theory. In addition, global numerical constraints (with respect to constants) can also be tested. It is proven emptiness decidability without a further complexity analysis. In this paper, besides showing decidability of a logic resulting from the addition of global numerical constraints to a alternation-free two-way $\mu$-calculus for trees, we provide an optimal satisfiability algorithm for the logic.

Contributions and Outline. We introduce in Section 2 an extension of the $\mu$-calculus (for trees) with global counting constructs called $\mu$ TLIN. These constructs restrict the number of nodes (with respect to binary constants) occurring in any region of the tree models.

In Section 3, we describe a useful application of $\mu$ TLIN in the context of XML. It is shown that an extension of XPath with counting constructs on multi-directional regular paths can be linearly embedded by the logic.

Analogously as in Section 3, we provide in Section 4 a linear embedding for regular tree languages (XML schemas) with counting constructs.

Section 5 is about succinctness. It is shown that the logic with global constraints is at least exponentially more succinct than the graded $\mu$-calculus.

Section 6 is devoted to show that the proposed logic is decidable. With this result we can thus use the logic as a reasoning framework for XPath queries with schema and counting constraints. However, the time complexity bound set for decidability is doubly exponential.

We improve the complexity bound for the logic in Section 8, It is described a satisfiability algorithm for the logic, and it is shown that the time complexity of the algorithm is single exponential. Before the description of the algorithm, we provide some preliminaries in Section 7. The complexity bound for the satisfiability algorithm, together with the linear embedding in Sections 3 and 4, imply EXPTIME characterizations of regular path queries (XPath) and regular tree languages (XML schemas) extended with global numerical constraints. Moreover, due to the fact that reasoning on regular tree languages is in 
EXPTIME-complete, the logic then represents an optimal reasoning framework for XPath queries and XML schemas with counting.

We conclude in Section 9 with a summary of the paper and a discussion of further research directions.

\section{A Modal Tree Logic with Global Numerical Constraints}

We consider through the paper labeled unranked trees. The tree logic with global numerical constraints $(\mu$ TLIN) is a modal tree logic (TL) with a least fixed-point $(\mu)$, inverse modalities (I), and global numerical constructs $(\mathrm{N})$. In contrast with graded modalities, where the number of nodes can be restricted only if they are immediate successors of a given node, the counting constructs in our logic can restrict the number of nodes occurring in any part of the tree model.

2.1. Syntax and semantics. In the context of tree models, modalities $m$ in modal formulas are defined by $M=\{\downarrow, \rightarrow, \uparrow, \leftarrow\} . \downarrow$ and $\rightarrow$ stand for the children and right sibling relations, respectively. $\uparrow$ and $\leftarrow$ are the corresponding inverse modalities, that is, the parent and left sibling relations. For a modality $m$, its inverse is written $\bar{m}$.

Definition 2.1 (Syntax). We define the set of $\mu$ TLIN formulas with the following grammar:

$$
\phi:=p|x| \neg \phi|\phi \vee \phi|\langle m\rangle \phi|\mu x . \phi| \phi>k
$$

Numerical constraints $k$ in counting formulas are assumed to be integer numbers in binary form. We use the following notation: $\phi \wedge \psi$ instead of $\neg(\neg \phi \vee \neg \psi)$, $\top$ instead of $\phi \vee \neg \phi$, and $\phi \leqslant k$ instead of $\neg(\phi>k)$. In the sequel, we often write counting formulas $\phi \# k$ for $\# \in\{\leqslant,>\}$. We define the size (length) of a formula $|\phi|$ as usual: $|p|=|x|=1$; $|\neg \phi|=|\langle m\rangle \phi|=|\mu x . \phi|=1+|\phi| ;|\phi \vee \psi|=1+|\phi|+|\psi| ;$ and $|\phi>k|=\log (k+1)+|\phi|$.

We consider the traditional assumption that variables can only occur in the scope of a modality or a counting operator. In addition, we assume variables do not occur in the scope of both, a modality and its converse. For instance, $\mu x .\langle\downarrow\rangle x \vee\langle\uparrow\rangle x$ is not allowed].

In a given tree, formulas are interpreted as subsets of tree nodes. Propositions serve as node labels. Negation is interpreted as set complement. Conjunctions and disjunctions are interpreted as the intersection and union of sets, respectively. Modal formulas $\langle m\rangle \phi$ are true in a node when there is an accessible node, through $m$, such that the formula $\phi$ holds. The $\mu$ operator is interpreted as a least fixpoint. The formula $\phi>k$ holds in every node of the tree model, if and only if, $\phi$ holds in at least $k+1$ nodes in the entire tree (see Definition 2.3).

We now give a formal description of the formula semantics. Finite tree structures are defined in the style of Kripke transition systems.

Definition 2.2 (Trees). A tree structure, or simply a tree, is a tuple $T=(\mathcal{P}, \mathcal{N}, \mathcal{R}, \mathcal{L})$, such that:

- $\mathcal{P}$ is the set of propositions;

- $\mathcal{N}$ is the finite set of nodes;

- $\mathcal{R}$ is a transition relation $(\mathcal{N} \times M) \times \mathcal{N}$ ( $M$ is the set of modalities $)$ forming a tree structure, we write $n^{\prime} \in \mathcal{R}(n, m)$ when $\left(n, m, n^{\prime}\right) \in \mathcal{R}$; and

\footnotetext{
${ }^{1}$ If variables do not occur in the scope of both, a modality and its converse, the greatest and least fixed-points coincide in the context of finite trees GLS07.
} 
- $\mathcal{L}$ is a left-total labeling relation on $\mathcal{N} \times \mathcal{P}$, written $p \in \mathcal{L}(n)$.

Definition 2.3 (Semantics). Given a tree $T$ and a valuation $V: \operatorname{Var} \mapsto 2^{\mathcal{N}}$, where $V a r$ is a fixed set of variables, the formula semantics is defined as follows:

$$
\begin{aligned}
& \llbracket p \rrbracket_{V}^{T}=\{n \mid p \in \mathcal{L}(n)\} \quad \llbracket x \rrbracket_{V}^{T}=V(x) \\
& \llbracket \neg \phi \rrbracket_{V}^{T}=\mathcal{N} \backslash \llbracket \phi \rrbracket_{V}^{T} \quad \llbracket \phi \vee \psi \rrbracket_{V}^{T}=\llbracket \phi \rrbracket_{V}^{T} \cup \llbracket \psi \rrbracket_{V}^{T} \\
& \llbracket\langle m\rangle \phi \rrbracket_{V}^{T}=\left\{n \mid \mathcal{R}(n, m) \cap \llbracket \phi \rrbracket_{V}^{T} \neq \varnothing\right\} \quad \llbracket \mu x . \phi \rrbracket_{V}^{T}=\bigcap\left\{\mathcal{N}^{\prime} \mid \llbracket \phi \rrbracket_{V\left[\mathcal{N}^{\prime} / x\right.}^{T} \subseteq \mathcal{N}^{\prime}\right\} \\
& \llbracket \phi>k \rrbracket_{V}^{T}= \begin{cases}\mathcal{N} & \text { if }\left|\llbracket \phi \rrbracket_{V}^{T}\right|>k \\
\varnothing & \text { otherwise }\end{cases}
\end{aligned}
$$

If the interpretation of a formula $\phi$ is not empty for a given tree $T$, i.e. $\llbracket \phi \rrbracket_{V}^{T} \neq \varnothing$, we say the tree $T$ satisfies the formula $\phi$. This is often written $T \models \phi$. A formula is said to be satisfiable if there is a tree satisfying it. Two formulas $\phi$ and $\psi$ are equivalent, if and only if, for every tree $T, T$ satisfies $\phi$, if and only if, $T$ satisfies $\psi$.

Example 2.4. We can express existential statements with counting formulas. For instance, if we want to select the nodes expressed by a formula $\psi$, only if there is a node satisfying $\phi$, then we write:

$$
(\phi>0) \wedge \psi
$$

Universality can also be expressed. The following formula selects the $\psi$ nodes when every node satisfies $\phi$ :

$$
[(\neg \phi) \leqslant 0] \wedge \psi
$$

Note that with counting formulas it is also possible to restrict the number of nodes occurring in a particular region. First, consider for instance the descendants region. This can be expressed as follows:

$$
\mu x \cdot\langle\uparrow\rangle\left(p_{0} \vee x\right)
$$

This formula denotes the descendants of the $p_{0}$ nodes. Recall that $\uparrow$ denotes the parent relation. Hence, the formula holds in nodes from where, by recursive navigations through parents, nodes named $p_{0}$ are accessible. Then, if we want to restrict the number of descendants of the $p_{0}$ nodes in a tree, then we write:

$$
\left[\mu x .\langle\uparrow\rangle\left(p_{0} \vee x\right)\right] \leqslant 6
$$

Now, if we want to restrict the number of some descendants, say descendants named $p_{1}$, then we write:

$$
\left(\left[\mu x .\langle\uparrow\rangle\left(p_{0} \vee x\right)\right] \wedge p_{1}\right) \leqslant 6
$$

Notice that $\mu x .\langle\uparrow\rangle\left(p_{0} \vee x\right) \wedge p_{1}$ holds in all $p_{1}$ descendants of each $p_{0}$ node. Hence, if in a model there are 2 nodes named $p_{0}$ with 2 and 3 descendants named $p_{1}$, respectively, then the formula $\left(\left[\mu x .\langle\uparrow\rangle\left(p_{0} \vee x\right) \vee\langle\leftarrow\rangle x\right] \wedge p_{1}\right) \leqslant 6$ holds due to all 6 descendants of both $p_{0}$ nodes (see Figure 10. However, one may also want to restrict the number of descendants of a particular node. This can be done by isolating the origin node from where navigation starts (during counting). For this purpose we first define the following formula:

$$
(o \leqslant 1) \wedge(o>0)
$$

In this formula, proposition $o$ occurs exactly once in a model. If we want to indentify where $o$ occurs, then we write:

$$
(o=1) \wedge o
$$




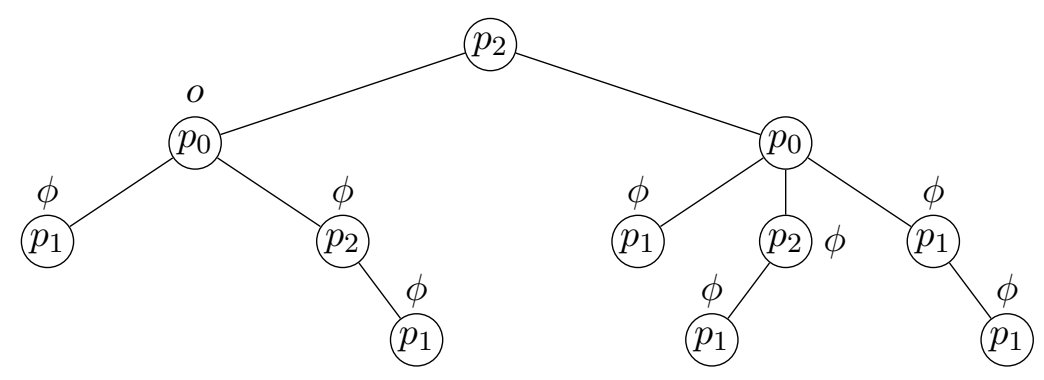

Figure 1: Tree model example: descendant region of $p_{0}$ nodes is denoted by the formula $\phi \equiv \mu x .\langle\uparrow\rangle\left(p_{0} \vee x\right)$; formula $\left(\phi \wedge p_{1}\right) \leqslant 6$ holds because the $p_{0}$ nodes have exactly 6 descendants labeled with $p_{1} ;\left(\mu x .\left[\left(p_{0} \wedge o \wedge o=1\right) \vee\langle\uparrow\rangle x\right] \wedge p_{1}\right) \leqslant 2$ is true because there is a $p_{0}$ node, the one marked with $o$, with 2 descendants named $p_{1}$.

where $o=1$ stands for $(o \leqslant 1) \wedge(o>0)$. Note that formula $(o=1) \wedge o$ selects an node only if the formula is true in exactly that node, then this formula can be seen as a nominal [BLMV06]. Now that we can isolate a single node in a model, we can thus restrict the counting from a particular node, consider for instance the following formula:

$$
\left[\mu x \cdot\langle\uparrow\rangle\left(\left[(o=1) \wedge o \wedge p_{0}\right] \vee x\right)\right] \leqslant 2
$$

This formula is true in models where there is single node with no more than 2 descendants. If in addition, we want to name the descendants, say $p_{1}$, then we write:

$$
\left[\mu x .\langle\uparrow\rangle\left(\left[(o=1) \wedge o \wedge p_{0}\right] \vee x\right) \wedge p_{1}\right] \leqslant 2
$$

A graphical respresentation of the examples above is depicted in Figure 1 .

\section{Counting Regular Path Queries}

The navigation core of the XPath query language (for XML documents) has been formalized as regular path queries, and it is known to correspond to $\mathrm{FOL}^{2}$ [CM09, Mar05. In this Section, we introduce an extension of regular path queries with counting constructs. In contrast with the counting extension of regular path queries reported in [BGLS11, where counting is limited to children paths only, the counting constructs described in this work are able to constrain arbitrary regular paths. We also provide in this Section a linear characterization of the counting extension of regular path queries into $\mu$ TLIN.

3.1. Syntax and semantics. We first describe the extension of regular paths with counting constructs on multi-directional paths. We call this extension CPath.

Definition 3.1 (Syntax). The syntax of CPath queries $\rho$ is given as follows:

$$
\begin{array}{ll}
\alpha:=\downarrow|\rightarrow| \uparrow|\leftarrow| \downarrow^{\star} \mid \uparrow^{\star} & \varrho:=\top|\alpha| p|\alpha: p| \varrho / \varrho \mid \varrho[\beta] \\
\beta:=\varrho>k|\beta \vee \beta| \neg \beta & \rho:=\varrho|/ \rho| \rho \cup \rho|\rho \cap \rho| \rho \backslash \rho
\end{array}
$$

where $p$ is a proposition, and $k$ is a positive integer in binary. 
We also consider the following syntatic sugar: $\varrho \leqslant k$ is written instead of $\neg(\varrho>k)$; $\varrho$ instead of $\varrho>0 ; \beta_{1} \wedge \beta_{2}$ instead of $\neg\left(\neg \beta_{1} \vee \neg \beta_{2}\right)$; and $\varrho\left[\beta_{1}\right]\left[\beta_{2}\right]$ instead of $\varrho\left[\beta_{1} \wedge \beta_{2}\right]$.

The CPath expressions are interpreted as node-selection queries on tree structures. In particular, the axis relations $\alpha$ are interpreted as follows: children $\downarrow$, following sibling $\rightarrow$, parent $\uparrow$, previous sibling $\leftarrow$, descendants $\downarrow^{\star}$, and ancestors $\uparrow^{\star}$. Step paths $\alpha: p$ selects the $p$ nodes reachable by $\alpha$. Symbol / is used to compose paths. A qualified path $\varrho[\beta]$ selects the nodes denoted by $\varrho$ that satisfies the boolean condition $\beta$. A qualified path $[\varrho>k]$ is true when $\varrho$ selects at least $k$ nodes. The boolean combination of qualifiers $\beta$ are interpreted in the obvious manner. The path $/ \rho$ selects the nodes denoted by $\rho$ that are reachable from the root. Union, intersection and difference of paths are interpreted as expected. Before given a formal description of the CPath semantics (inspired from [tCM09]), we introduce the following notation: in a Kripke structure, $n_{1} \stackrel{\alpha}{\rightarrow} n_{2}$ means than $n_{1}$ is related by means of $\alpha$ with $n_{2}$, where $\alpha$ can be any axis relation $\left(\downarrow, \rightarrow, \uparrow, \leftarrow, \downarrow^{\star}, \uparrow^{\star}\right)$.

Definition 3.2 (Semantics). The semantics of CPath queries is defined by a function $\llbracket \cdot \rrbracket$ from CPath queries with respect to a tree $T$, to pairs of nodes in $T$.

$$
\begin{aligned}
& \llbracket \top \rrbracket^{T}=\mathcal{N} \times \mathcal{N} \\
& \llbracket p \rrbracket^{T}=\{(n, n) \mid p \in \mathcal{L}(n)\} \\
& \llbracket \alpha \rrbracket^{T}=\left\{\left(n_{1}, n_{2}\right) \mid n_{1} \stackrel{\alpha}{\rightarrow} n_{2}\right\} \\
& \llbracket \alpha: p \rrbracket^{T}=\left\{\left(n_{1}, n_{2}\right) \in \llbracket \alpha \rrbracket^{T} \mid p \in \mathcal{L}\left(n_{2}\right)\right\} \\
& \llbracket \varrho_{1} / \varrho_{2} \rrbracket^{T}=\llbracket \varrho_{1} \rrbracket^{T} \circ \llbracket \varrho_{2} \rrbracket^{T} \\
& \llbracket \varrho[\beta] \rrbracket^{T}=\left\{\left(n_{1}, n_{2}\right) \in \llbracket \varrho \rrbracket^{T} \mid n_{2} \in \llbracket \beta \mathbb{\rrbracket}^{T}\right\} \\
& \llbracket \varrho>k \mathbb{\rrbracket}^{T}=\left\{n_{1}||\left\{n_{2} \mid\left(n_{1}, n_{2}\right) \in \llbracket \varrho \rrbracket^{T}\right\} \mid>k\right\} \\
& \mathbb{\amalg} \neg \beta \mathbb{\rrbracket}^{T}=\mathcal{N} \backslash \mathbb{\|} \beta \mathbb{\rrbracket}^{T} \\
& \llbracket \beta_{1} \vee \beta_{2} \mathbb{\rrbracket}^{T}=\llbracket \beta_{1} \mathbb{\rrbracket}^{T} \cup \llbracket \beta_{2} \mathbb{\rrbracket}^{T} \\
& \llbracket / \varrho \rrbracket^{T}=\left\{(r, n) \in \llbracket \varrho \rrbracket^{T} \mid r \text { is the root }\right\} \\
& \llbracket \rho_{1} \cup \rho_{2} \rrbracket^{T}=\llbracket \rho_{1} \rrbracket^{T} \cup \llbracket \rho_{2} \rrbracket^{T} \\
& \llbracket \rho_{1} \cap \rho_{2} \rrbracket^{T}=\llbracket \rho_{1} \rrbracket^{T} \cap \llbracket \rho_{2} \rrbracket^{T} \\
& \llbracket \rho_{1} \backslash \rho_{2} \rrbracket^{T}=\llbracket \rho_{1} \rrbracket^{T} \backslash \llbracket \rho_{2} \rrbracket^{T}
\end{aligned}
$$

Notice that the function $\mathbb{\|} \cdot \mathbb{I}^{\cdot}$ is introduced to distinguish the interpretation of paths inside qualifiers.

Example 3.3. Consider for instance the following composition of paths:

$$
\uparrow^{\star}: p_{1} / \downarrow^{\star}: p_{2}
$$

This query, evaluated from some context (a node subset), navigates to the $p_{1}$ ancestors of the context, and from there, it selects the $p_{2}$ descendants. Now consider the following qualified path:

$$
\uparrow^{\star}: p_{1}\left[\downarrow^{\star}: p_{2}\right]
$$

In constrast with the previous example, this query selects the $p_{1}$ ancestors with at least 1 descendant named $p_{2}$.

Proposition 3.4 (Succinctness). For any tree $T$ and CPath expression $\rho$, there is a regular path (CPath without counting) $\rho^{\prime}$, such that

- $\llbracket \rho \rrbracket^{T}=\llbracket \rho^{\prime} \rrbracket^{T}$, and

- the size of $\rho^{\prime}$ is exponentially greater than the size of $\rho$.

Proof. Given an expression $\varrho>k(k>0)$, we will show that that there is an equivalent path expression $\varrho^{\prime}$ without counting. We proceed by induction on the structure of $\varrho$. 
For the base cases, we consider the following replacements:

$$
\begin{aligned}
& \downarrow: p[\overbrace{\rightarrow: p[\rightarrow: p[\ldots]]}^{k \text { times }}] \text { instead of } \downarrow: p>k ;\overbrace{\rightarrow: p[\rightarrow: p[\ldots]]}^{k+1 \text { times }}] \text { instead of } \rightarrow: p>k ; \\
&\overbrace{\leftarrow: p[\leftarrow: p[\ldots]]}^{k+1 \text { times }}] \text { instead of } \leftarrow: p>k ; \\
& \downarrow^{\star}: p[\overbrace{\downarrow^{\star} \rightarrow: p\left[\downarrow^{\star} \rightarrow: p[\ldots]\right]}^{k \text { times }} \text { instead of } \downarrow^{\star}: p>k ; \text { and } \\
& \uparrow^{\star}: p[\overbrace{\uparrow^{\star} \rightarrow: p\left[\downarrow^{\star} \rightarrow: p[\ldots]\right]}^{k \text { times }} \text { instead of } \uparrow^{\star}: p>k ;
\end{aligned}
$$

where $\downarrow^{\star} \rightarrow: p[\beta]$ and $\uparrow^{\star} \rightarrow: p[\beta]$ are syntactic sugar for $\downarrow^{\star}: p[\beta] \vee \rightarrow: p[\beta]$ and $\uparrow^{\star}: p[\beta] \vee \rightarrow$ : $p[\beta]$, respectively.

Consider now the case $\left(\varrho_{1} / \varrho_{2}\right)>k$, this expression is replaced by $\varrho_{1}^{\prime} / \varrho_{2}^{\prime}$, where by induction we know that $\varrho_{1}^{\prime}$ and $\varrho_{2}^{\prime}$ are the counting-free expressions equivalent to $\varrho_{1}$ and $\varrho_{2}>k$, respectively.

Expression $\varrho_{1}\left[\varrho_{2}>k_{2}\right]>k_{1}$ is replaced by $\varrho_{1}^{\prime}\left[\varrho_{2}^{\prime}\right]$, such that by induction $\varrho_{1}^{\prime}$ and $\varrho_{2}^{\prime}$ are the counting-free expressions equivalent to $\varrho_{1}>k_{1}$ and $\varrho_{2}>k_{2}$, respectively.

Cases $\varrho\left[\beta_{1} \vee \beta_{2}\right]$ and $\varrho[\neg \beta]$ are also immediate by induction.

In the replacement described above, notice that numerical restrictions (in binary) are replaced by explicit path occurrences, it is hence easy to see the exponential blow-up in the size of the counting-free expression.

Definition 3.5 (Reasoning problems). We define the emptiness, contaiment and equivalence problems of CPath queries as follows.

- We say a query $\rho$ is empty, if and only if, for every tree $T$, its interpretation is empty, that is, $\llbracket \rho \rrbracket^{T}=\varnothing$;

- It is said that a query $\rho_{1}$ is contained in a query $\rho_{2}$, if and only if, for every tree $T$, each pair of nodes in the interpretation of $\rho_{1}$ is in the intepretation of $\rho_{2}$, that is, $\llbracket \rho_{1} \rrbracket^{T} \subseteq \llbracket \rho_{2} \rrbracket^{T}$; and

- Two queries $\rho_{1}$ and $\rho_{2}$ are equivalent, if and only if, for every tree $T, \rho_{1}$ is contained in $\rho_{2}$ and the other way around, that is, $\llbracket \rho_{1} \rrbracket^{T} \subseteq \llbracket \rho_{2} \rrbracket^{T}$ and $\llbracket \rho_{2} \rrbracket^{T} \subseteq \llbracket \rho_{1} \rrbracket^{T}$.

3.2. Logic characterization. Regular path queries (without counting) can be written in terms of the $\mu$-calculus [BGLS11]. For instance, the query $\downarrow^{\star}: p$, evaluated in the root $r$, selects the $p$ descendants of $r$. This can be written as follows:

$$
[\mu x .\langle\uparrow\rangle(r \vee x)] \wedge p
$$

If we want to evaluate the query in another context (node subset), represented by a $C$ formula, then we simply replace the occurrence of $r$ by $C$. For instance, let us say the context represented by all the nodes named $p_{0}$, then the $p$ ancestors of $p_{0}$ nodes can be written as follows:

$$
\left[\mu x .\langle\uparrow\rangle\left(p_{0} \vee x\right)\right] \wedge p
$$


In [BGLS11, it was also shown that an extension of regular path queries with counting on children paths can be expressed in terms of the two-way graded $\mu$-calculus. Children paths are of the forms $\downarrow: p$ and $\downarrow: p[\varrho]$. In this paper, we show that the $\mu$ TLIN counting constructs can describe more general counting constructs on arbitrary regular path queries, such as $\downarrow^{\star}: p_{1} / \uparrow^{\star}: p_{2}[\varrho]$.

Definition 3.6 (CPath queries into $\mu$ TLIN formulas). Given a context formula $C$, the translation $F$ from CPath queries into $\mu$ TLIN formulas is defined as follows:

$$
\begin{array}{ll}
F(\downarrow, C)=\langle\uparrow\rangle C & F(\rightarrow, C)=\langle\leftarrow\rangle C \\
F(\uparrow, C)=\langle\downarrow\rangle C & F(\leftarrow, C)=\langle\rightarrow\rangle C \\
F\left(\downarrow^{\star}, C\right)=\mu x .\langle\uparrow\rangle(C \vee x) & F\left(\uparrow^{\star}, C\right)=\mu x \cdot\langle\downarrow\rangle(C \vee x) \\
F(\alpha: p, C)=F(\alpha, C) \wedge p & F\left(\varrho_{1} / \varrho_{2}, C\right)=F\left(\varrho_{2}, F\left(\varrho_{1}, C\right)\right) \\
F(\varrho[\beta], C)=F(\varrho, C) \wedge o \wedge F(\beta,[o=1] \wedge o) & F(\varrho>k, C)=F(\varrho, C)>k \\
F(\neg \beta, C)=F^{\prime}(\beta, C) & F\left(\beta_{1} \vee \beta_{2}, C\right)=F\left(\beta_{1}, C\right) \vee F\left(\beta_{2}, C\right) \\
F(/ \varrho, C)=F(\varrho, C \wedge \neg(\langle\uparrow\rangle \top \wedge\langle\leftarrow\rangle \top)) & F\left(\rho_{1} \cap \rho_{2}, C\right)=F\left(\rho_{1}, C\right) \wedge F\left(\rho_{2}, C\right) \\
F\left(\rho_{1} \cup \rho_{2}, C\right)=F\left(\rho_{1}, C\right) \vee F\left(\rho_{2}, C\right) & F\left(\rho_{1} \backslash \rho_{2}, C\right)=F\left(\rho_{1}, C\right) \wedge F^{\prime}\left(\rho_{2}, C\right)
\end{array}
$$

where

$$
\begin{aligned}
& F^{\prime}(\rho)= \begin{cases}F^{\prime}(\varrho, C \wedge \neg[\langle\uparrow\rangle \uparrow \wedge\langle\leftarrow\rangle \top]) & \text { if } \rho \text { has the form } / \varrho, \\
\neg F(\rho) & \text { otherwise. }\end{cases} \\
& F^{\prime}(\varrho)= \begin{cases}\neg F\left(\varrho^{\prime}, C\right) \vee[o \wedge \neg F(\beta,[o=1] \wedge o)] & \text { if } \varrho \text { has the form } \varrho^{\prime}[\beta], \\
\neg F(\varrho) & \text { otherwise. }\end{cases}
\end{aligned}
$$

In general $F^{\prime}$ represent the negation of $F$, however in the case where there is a counting operator, the fresh proposition $o$, which serves to fix an origin node, is not negated. Note that the constraint $o=1 \wedge o$ is not affected by negation because it always occur in the scope of a counting operator.

Example 3.7. Consider the following query evaluated in a context $C$ :

$$
\downarrow: p_{1}\left[\downarrow^{\star}: p_{2}>k\right]
$$

The query selects the $p_{1}$ children of $C$ with at least $k+1$ descendants named $p_{2}$. The first part of the query $\downarrow: p_{1}$ is translated as follows:

$$
p_{1} \wedge\langle\uparrow\rangle C
$$

That is, the $p_{1}$ nodes with $C$ as parent. The translation of the counting expression $\downarrow^{\star}: p_{2}>k$ is

$$
o \wedge\left[p_{2} \wedge \mu x \cdot\langle\uparrow\rangle([o=1 \wedge o] \vee x)\right]>k
$$

This formula holds, if and only if, there are more than $k$ descendant nodes, named $p_{2}$, of a single node named $o$. Then, the translation of the entire query is the following:

$$
F\left(\downarrow: p_{1}\left[\downarrow^{\star}: p_{2}>k\right]\right)=\left(p_{1} \wedge\langle\uparrow\rangle C\right) \wedge\left(o \wedge\left[p_{2} \wedge \mu x .\langle\uparrow\rangle([o=1 \wedge o] \vee x)\right]>k\right)
$$

The proposition $o$ is used to fix a context for the counting subformula. $o$ holds in a single $p_{1}$ node, then the $p_{2}$ descendants of that particular $p_{1}$ node are the only ones counted. 
With the translation function $F$, we can now use the logic as a reasoning framework to solve emptiness, containment and equivalence of CPath queries, moreover, since translation $F$ does not introduce duplications, it is easy to see that the formula resulting from the translation has linear size with respect to the input query.

Theorem 3.8 (Query reasoning). For any CPath queries $\rho, \rho_{1}, \rho_{2}$, tree $T$ and valuation $V$, the following holds:

- $\llbracket \rho \rrbracket^{T}=\varnothing$ if and only if $\llbracket F(\rho, \top) \rrbracket_{V}^{T}=\varnothing$;

- $\llbracket \rho_{1} \rrbracket^{T} \subseteq \llbracket \rho_{2} \rrbracket^{T}$ if and only if $\llbracket F\left(\rho_{1}, \top\right) \wedge F^{\prime}\left(\rho_{2}, \top\right) \rrbracket_{V}^{T}=\varnothing$; and

- $F(\rho, \top)$ has linear size with respect to $\rho$ and $F^{\prime}\left(\rho_{1}, \top\right) \wedge F\left(\rho_{2}, \top\right)$ has linear size with respect to $\rho_{1}$ and $\rho_{2}$.

Proof. For the first item, we proceed by structural induction on $\rho$.

In order to proof the case when $\rho$ has the form $\varrho$, we will proof the following: $\varrho$ evaluated in a context $C$ is satisfiable by a tree $T$, if and only if, $F(\varrho, C)$ is satisfiable by $T$.

Consider $\rho$ is the basic query $\downarrow^{\star}: p$, then $F\left(\downarrow^{\star}: p, C\right)=p \wedge \mu x \cdot\langle\uparrow\rangle(C \vee x)$, which clearly selects exactly the same nodes than $\rho$ evaluated in $C$. The proof for the cases with the other axes $\left(\downarrow, \uparrow, \rightarrow, \leftarrow, \uparrow^{\star}\right)$ is similar.

Now let the input query be a composition of paths, that is, $\rho$ has the form $\varrho_{1} / \varrho_{2}$. Intuitively, $\varrho_{1} / \varrho_{2}$ selects the nodes denoted by $\rho_{2}$ evaluated from the nodes satisfying $\varrho_{1}$, that is, $\varrho_{1}$ is the context. That is precisely what it means $F\left(\varrho_{2}, F\left(\varrho_{1}, C\right)\right)$. By induction $F\left(\varrho_{1}, C\right)$ corresponds to $\varrho_{1}$, and then also by induction $F\left(\varrho_{2}, F\left(\varrho_{1}, C\right)\right)$ corresponds $\varrho_{1} / \varrho_{2}$ evaluated in $C$.

Before proving the case when the input query has the form $\varrho_{1}\left[\varrho_{2}>k\right]$, we need first to proof that $\varrho_{2}>k$ is satisfiable by $T$, if and only if, $F\left(\varrho_{2}, o=1 \wedge o\right)>k$ is satisfiable by $T$. This is achieved by induction on the structure of $\varrho_{2}$. Consider $\varrho_{2}$ has the form $\downarrow: p$. Then $F(\downarrow: p, \top)=p \wedge\langle\uparrow\rangle \top$. This formula selects all the $p$ children of the model. However according to the semantics of CPath queries (Definition 3.2), we need to count the $p$ children of a single node. This is achieved by fixing the context with a new fresh proposition o occurring only once in the model $o=1$. Hence $[p \wedge\langle\uparrow\rangle([o=1] \wedge o)]>k$ is satisfiable by $T$, if and only if, $\downarrow: p>k$ is satisfiable by $T$. We proceed analogously for the other axes. For the other cases of $\varrho_{2}$, that is, when $\varrho_{2}$ is a composition of paths $\left(\varrho_{2}^{\prime} / \varrho_{2}^{\prime \prime}\right)$ and a qualified path $\left(\varrho_{2}^{\prime}\left[\beta^{\prime}\right]\right)$, the proof goes straightforward by induction.

Now that we know that $\varrho_{2}>k$ is satisfiable by $T$, if and only if, $F\left(\varrho_{2}\right)>k$ is satisfiable by $T$, and that by induction, $\varrho_{1}$ evaluated in $C$ is satisfiable by $T$, if and only if, $F\left(\varrho_{1}, C\right)$ is satisfiable $T$, we can thus infer that $F\left(\varrho_{1}, C\right) \wedge o \wedge F\left(\varrho_{2},[o=1] \wedge o\right)$ is satisfiable by $T$, if and only if, $\varrho_{1}\left[\varrho_{2}>k\right]$ is satisfiable in context $C$ by $T$. Note that $o$ is used to select a single $\varrho_{1}$ node.

When $\varrho$ has the form $\varrho_{1}[\beta]$, the cases when $\beta$ is a disjunction or a negation are immediate by induction. In the case of negation, it is important to notice that the negation of $F\left(\varrho^{\prime},[o=\right.$ 1] $\wedge o)>k$ does not affect the context, that is, negation never goes inside the formula $[o=1] \wedge o$.

Consider now the case when the input query has the form $\rho_{1} \backslash \rho_{2}$. The only interesting case is when $\rho_{2}$ has the form $\varrho_{1}\left[\varrho_{2}>k\right]$. It is easy to see, by induction, that $F\left(\rho_{1}, C\right)$ is satisfiable by $T$, if and only if, $\rho_{1}$ is satisfiable by $T$. Also by induction we also know that $\neg F\left(\varrho_{1}, C\right) \vee\left(o \wedge \neg F\left(\varrho_{2},[o=1] \wedge o\right)\right)$ is satisfiable by $T$, if and only if, $\varrho_{1}\left[\varrho_{2}>k\right]$ is not satisfiable by $T$. We can hence conclude that $F\left(\rho_{1}, C\right) \wedge\left[\neg F\left(\varrho_{1}, C\right) \vee\left(o \wedge \neg F\left(\varrho_{2}[o=1] \wedge o\right)\right)\right]$ is satisfiable by $T$, if and only if, $\rho_{1} \backslash\left(\varrho_{1}\left[\varrho_{2}>k\right]\right)$ also does. 
The cases when the input query has the forms $\rho_{1} \cup \rho_{2}, \rho_{1} \cap \rho_{2}$, and $/ \rho_{1}$ are straightforward by induction.

For the second item, we proceed analogously as in the first item in the case when the input query has the form $\rho_{1} \backslash \rho_{2}$.

The third item is proven immediately by structural induction on the input query and by noticing that function $F$ does not introduce duplications.

\section{Regular Tree Languages with Counting}

Regular tree language expressions (types, schemas) can be seen as the arborescent version of regular expressions. These expressions are used to describe sets of trees, and they encompass most common XML schema languages, such as DTDs, XML schema and RelaxNG MLMK05. Consider for instance the following expression:

$$
p_{1}\left[p_{2}^{\star}\right]
$$

This expression is interpreted as the set of trees (XML documents) rooted by $p_{1}$ with 0 or more contiguous children named $p_{2}$.

In this paper, we consider an extension of regular tree languages with counting constructs. These constructs serve to constrain the number of children occurrence. For example, if one wants to describe the trees rooted by $p_{1}$ with at most 5 children named $p_{2}$, one may write:

$$
p_{1}\left[p_{2}^{\leqslant 5}\right]
$$

4.1. Syntax and semantics. We now give a precise definition of the regular tree types with counting.

Definition 4.1 (CTypes syntax). The syntax of CTypes expressions is defined by:

$$
e:=\epsilon|x| e \cdot e|e+e| \text { let } \bar{x} \cdot \bar{e} \text { in } e\left|p\left[e^{>k}\right]\right| p\left[e^{\leqslant k}\right]
$$

We often write $p[e]$ instead of $p[e>0]$. Variables cannot occur free, that is, variables always occur under the scope of a fixpoint operator.

$\epsilon$ is used for the empty tree. Concatenation and alternation are expressed as usual with the respective symbols $\cdot$ and + . The binder is used for recursion. The Kleene star and other common notation for regular languages are defined as follows: $e^{\star}=$ let $x .(e \cdot x)+\epsilon$ in $x$, $e^{+}=e \cdot e^{\star}$, and $e^{?}=\epsilon+e$. Counting expressions $p\left[e^{\# k}\right]$ denote the set of trees rooted at $p$ such that the number of children subtrees matching with $e$ satisfy the numerical constraint $\# k$. In contrast with other forms of counting in regular tree languages [Gel10], we do not force the counted nodes to be contiguous siblings.

Definition 4.2 (CTypes semantics). Given a valuation $V$ into trees, the interpretation of CTypes expressions is given as follows:

$$
\begin{array}{ll}
\llbracket \epsilon \rrbracket_{V}=\{\varnothing\} & \llbracket x \rrbracket_{V}=V(x) \\
\llbracket e_{1} \cdot e_{1} \rrbracket_{V}=\llbracket e_{1} \rrbracket_{V} \cdot \llbracket e_{2} \rrbracket_{V} & \llbracket e_{1}+e_{2} \rrbracket_{V}=\llbracket e_{1} \rrbracket_{V} \cup \llbracket e_{2} \rrbracket_{V} \\
\llbracket \text { let } \bar{x} . \bar{e} \text { in } e \rrbracket_{V}=\llbracket e \rrbracket_{\mathrm{lfp}(V)} & \llbracket p\left[e^{\# k}\right] \rrbracket_{V}=\{T \mid \text { the root of } T \text { is labeled by } p \text { and the number } \\
& \text { of children subtrees in } \left.\llbracket e \rrbracket_{T} \text { satisfies } \# \mathrm{k}\right\}
\end{array}
$$


where $\operatorname{lfp}(f)$ is the least fixpoint of $f$ defined $\operatorname{lfp}\left(V^{\prime}\right)=V\left[\bar{x} / \overline{\llbracket e \rrbracket_{V^{\prime}}}\right]$. Note that $V$ is monotone according to subset ordering, hence it always has a fixpoint due to the Fixpoint Theorem Tar55].

It was shown in [BGLS11] that any CTypes expression can be written in terms of $\mu$ calculus formulae. We also know that the graded $\mu$-calculus is as expressive as the plain $\mu$-calculus [Bar11. It is also well-known that the plain $\mu$-calculus and regular tree languages (types) are equally expressive [JW96]. It is then easy to see that counting operators (CTypes) do not introduce more expressive power in regular tree languages. Also, by Theorem 6.3. we can conclude that $\mu$ TLIN and CTypes are equally expressive.

4.2. Logic characterization. CTypes without counting can be linearly characterized by the simple $\mu$-calculus [BGLS11. Moreover, in the same work it is also shown that the counting constructs of CTypes can be captured by the graded $\mu$-calculus. We now show that $\mu$ TLIN can also capture CTypes expression and hence be used as a reasoning framework. For instance, the above example $p_{1}\left[p_{2}^{\star}\right]$ can be expressed as follows:

$$
p_{1} \wedge\left(\neg\langle\downarrow\rangle \top \vee\langle\downarrow\rangle\left[\neg\langle\leftarrow\rangle \top \wedge \mu x . p_{2} \wedge(\langle\rightarrow\rangle x \vee \neg\langle\rightarrow\rangle \top)\right]\right)
$$

We now give a general translation function.

Definition 4.3 (CTypes expressions into $\mu$ TLIN formulas). The translation function $F$ from CTypes expressions to $\mu$ TLIN formulas is given as follows:

$$
\begin{array}{ll}
F(\epsilon)=\neg \top & F\left(e_{1}+e_{2}\right)=F\left(e_{1}\right) \cup F\left(e_{2}\right) \\
F\left(e_{1} \cdot e_{2}\right)=F\left(e_{1}\right) \wedge\left\langle\rightarrow F\left(e_{2}\right)\right. & F(\text { let } \bar{x} . \bar{e} \text { in e })=\mu \bar{x} \cdot \overline{F(e)} \text { in } F(e) \\
F\left(p\left[e^{\# k}\right]\right)=p \wedge o \wedge(F(e) \wedge\langle\uparrow\rangle[o=1 \wedge o]) \# k
\end{array}
$$

Formula $\mu \bar{x} . \bar{\phi}$ in $\phi$ is a generalization of the least fixpoint. Its formal semantics is defined as follows:

$$
\llbracket \mu \bar{x} . \bar{\phi} \text { in } \phi \rrbracket_{V}^{T}=\llbracket \phi \rrbracket_{V\left[\overline{N^{\prime \prime}} / \bar{x}\right]}^{T}, \text { where } \overline{N^{\prime \prime}}=\bigcap\left\{\overline{N^{\prime}} \mid \overline{\llbracket \phi \rrbracket_{V\left[\overline{N^{\prime}} / \bar{x}\right.}^{T}} \subseteq \overline{N^{\prime}}\right\} .
$$

Note that this generalization does not provide more expressive power and it is only used for a succinct translation of his analogous operator in CTypes expressions.

Now consider an example for the translation function. The expression above $p_{1}\left[p_{2}^{\leqslant} 5\right]$ is translated as follows:

$$
F\left(p_{1}\left[p_{2}^{\leqslant k}\right]\right)=p_{1} \wedge o \wedge\left(p_{2} \wedge\langle\uparrow\rangle[o=1 \wedge o]\right) \leqslant 5
$$

Notice that the fresh proposition $o$ is used to count from a fixed context in an analogous manner as done for regular path queries. It is then necessary to define a safe negation for the translation $F$ in order to properly model the containment and equivalence of CTypes expressions. Safe negation of $F$ is defined by $F^{\prime}$ as follows.

Definition 4.4. We define the following translation function from CTypes expressions into $\mu$ TLIN formulas.

$F^{\prime}(e)= \begin{cases}\neg p \vee\left(o \wedge \neg\left[\left(F\left(e_{0}\right) \wedge \mu x .\langle\uparrow\rangle[o=1 \wedge o] \vee\langle\leftarrow\rangle x\right) \# k\right]\right) & \text { if } e \text { has the form } p\left[e_{0}^{\# k}\right], \\ \neg F(e) & \text { otherwise. }\end{cases}$ 
We can now define the reasoning problems of CTypes expressions in terms of $\mu$ TLIN formulas.

Theorem 4.5 (CTypes reasoning). For any CTypes expressions $e, e_{1}$ and $e_{2}$, tree $T$ and valuation $V$, we have that:

- $\llbracket e \rrbracket_{V}=\varnothing$, if and only if, $\llbracket F(e) \rrbracket_{V}^{T}=\varnothing$;

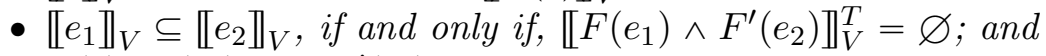

- $F(e), F\left(e_{1}\right)$ and $F^{\prime}\left(e_{2}\right)$ have linear size with respect to $e, e_{1}$ and $e_{2}$, respectively.

Proof. The proof goes by structural induction on the input CTypes expressions in an analogous manner as the proof of Theorem 3.8. We will only show the case when the CTypes expression has the form $p\left[e^{\# k}\right]$ for the first item. By induction we know $F(e)$ is satisfiable by a tree $T$, if and only if, $e$ is satisfiable. Then the formula $[F(e) \wedge\langle\uparrow\rangle(o=1 \wedge o)] \# k$ is satisfiable by $T$, if and only if, there is a node with children matching $F(e)$ and satisfying the numerical constraint \#k. Therefore $p \wedge o \wedge[F(e) \wedge\langle\uparrow\rangle(o=1 \wedge o)] \# k$ is satisfiable by $T$, if and only if, $p\left[e^{\# k}\right]$ is satisfiable by $T$.

\section{SuCCINCTENESS}

We show in this Section that $\mu$ TLIN is at least exponentially more succinct that the graded $\mu$-calculus BLMV06. This is done via a GCTL embedding. We know from Bianco et al. BMM10, BMM12 that the Graded Computation Tree Logic (GCTL) is at least exponentially more succinct than the graded $\mu$-calculus. We then describe a linear embedding of GCTL into $\mu$ TLIN. A precise definition of GCTL formulas is first given.

Definition 5.1 (Syntax). The set of Graded Computation Tree Logic formulas is inductively defined by the following grammar.

$$
\phi:=p|\neg \phi| \phi \vee \phi\left|E^{>k} X \phi\right| E^{>k} G \phi \mid E^{>k} \phi U \phi
$$

Formulas are also interpreted as node subsets of finite tree structures. Proposition are also used as node labels, and the boolean operators are interpreted as expected. Formula $E^{>k} X \phi$ is true in nodes with more than $k$ children where $\phi$ holds. $E^{>k} G \phi$ holds in nodes with more than $k$ downward paths leading to a leaf, such that $\phi$ is true in each path node. And formula $E^{>k} \phi U \psi$ holds in nodes $n_{0}$ with more than $k$ downward paths $n_{0}, \ldots, n_{k}$, such that $\psi$ holds in $n_{k}$ and $\phi$ is true in $n_{i}$ for every $i<k$.

The all but graded operator $A^{\leqslant k}$ is defined as follows:

$$
\begin{aligned}
& A^{\leqslant k} X \phi \equiv \neg E^{>k} X \neg \phi, \quad A^{\leqslant k} G \phi \equiv \neg E^{>k} F \neg \phi, \\
& E^{>k} F \phi \equiv E^{>k} \top U \phi, \quad A^{\leqslant k} \phi U \psi \equiv \bigvee_{k_{1}+k_{2}=k} \neg\left(E^{>k_{1}}[\neg \psi U(\neg \phi \wedge \neg \psi)] \vee E^{>k_{2}} G \neg \psi\right) .
\end{aligned}
$$

$A^{\leqslant k} X \phi$ selects nodes with at most $k$ children where $\phi$ does not hold; $A^{\leqslant k} G \phi$ restricts to at most $k$ the number of downward paths leading to a leaf, such that $\phi$ does not hold in each path node; $E^{>k} F \phi$ counts at least $k$ paths where $\phi$ holds at least once; and $A^{\leqslant k} \phi U \psi$ constrains to at most $k$ the number of downward paths such that the following does not hold: $\phi$ and $\neg \psi$ are true and in each path node except the last one where $\psi$ is true.

In order to give a precise GCTL semantics we first describe some useful notations about downward paths. 
Definition 5.2 (Children path). Given a tree structure $T$, a children path $\alpha_{n_{0}}^{n_{k}}$ starting at node $n_{0}$ and ending at node $n_{k}$ is a finite set of nodes $\left\{n_{0}, n_{1}, \ldots, n_{k}\right\}$, such that $n_{i+1} \in$ $\mathcal{R}\left(n_{i}, \downarrow\right)$ for $i=0, \ldots, k$. If the ending node $n_{k}$ is a leaf, that is, $\mathcal{R}\left(n_{k}, \downarrow\right)=\varnothing$, then we may avoid to write the ending node $\alpha_{n_{0}}$. If the starting and the ending node is the same, then the path is defined as the singleton $\alpha_{n}^{n}=\{n\}$.

Definition 5.3 (Semantics). Given a tree structure $T$, the interpretation of GCTL formulas is given as follows.

$$
\begin{aligned}
\llbracket p \rrbracket^{T} & =\{n \in \mathcal{L}(p)\} \\
\llbracket \neg \phi \rrbracket^{T} & =\mathcal{N} \backslash \llbracket \phi \rrbracket^{T} \\
\llbracket \phi \vee \psi \rrbracket^{T} & =\llbracket \phi \rrbracket_{V}^{T} \cup \llbracket \psi \rrbracket_{V}^{T} \\
\llbracket E^{>k} X \phi \rrbracket^{T} & =\left\{n|| \mathcal{R}(n, \downarrow) \cap \llbracket \phi \rrbracket^{T} \mid>k\right\} \\
\llbracket E^{>k} G \phi \rrbracket^{T} & =\left\{n||\left\{\alpha_{n} \mid \alpha_{n} \subseteq \llbracket \phi \rrbracket^{T}\right\} \mid>k\right\} \\
\llbracket E^{>k} \phi U \psi \rrbracket^{T} & =\left\{n_{0}||\left\{\alpha_{n_{0}}^{n_{k}} \neq \varnothing \mid n_{k} \in \llbracket \psi \rrbracket^{T}, \alpha_{n_{0}}^{n_{k-1}} \subseteq \llbracket \phi \rrbracket^{T}\right\} \mid>k\right\}
\end{aligned}
$$

As expected, GCTL formulas can be described in terms of $\mu$ TLIN formulas. We now give a precise definition of this embedding.

Definition 5.4 (GCTL embedding). The function $F$ from GCTL formulas to $\mu$ TLIN formulas is defined as follows:

$$
\begin{array}{ll}
F(p)=p & F(\neg \phi)=\neg F(\phi) \\
F(\phi \vee \psi)=F(\phi) \vee F(\psi) & F\left(E^{>k} X \phi\right)=o \wedge(F(\phi) \wedge\langle\uparrow\rangle[o \wedge o=1])>k \\
\left.F\left(E^{>k} G \phi\right)=o \wedge(\neg\langle\downarrow\rangle\rceil \wedge \mu x . F(\phi) \wedge[\langle\uparrow\rangle x \vee(o \wedge o=1)]\right)>k \\
F\left(E^{>k} \phi U \psi\right)=o \wedge(\psi \wedge[o \vee\langle\uparrow\rangle \mu x . F(\phi) \wedge(\langle\uparrow\rangle x \vee[o \wedge o=1])])>k
\end{array}
$$

Theorem 5.5 (Embedding). For any GCTL formula $\phi$, tree $T$ and valuation $V$, we have that:

$$
\llbracket \phi \rrbracket^{T} \neq \varnothing \text { if and only if } \llbracket F(\phi) \rrbracket_{V}^{T} \neq \varnothing
$$

and $F(\phi)$ has linear size with respect to $\phi$.

Proof. By induction on the structure of the input formula.

The base case, when the formula is a proposition, is trivial. The cases of disjunction and negation are immediate by induction.

Consider now the case when the input formula has the form $E^{>k} X \phi$. By induction we know that $\phi$ is satisfiable by $T$, if and only if, $F(\phi)$ also does. Now, it is easy to see that $F(\phi) \wedge\langle\uparrow\rangle \top$ selects all the children nodes where $\phi$ is true. Then $(F(\phi) \wedge\langle\uparrow\rangle[o \wedge o=1])>k$ is true when the single node marked by $o$ has more than $k$ children where $\phi$ holds. Therefore $F\left(E^{>k} X \phi\right)$ is satisfiable by $T$, if and only if, $E^{>k} X \phi$ also does.

Consider now the case for $E^{>k} G \phi$. By induction we know that $T$ satisfies $\phi$, if and only if, $T$ also satisfies $F(\phi)$. Now, recall that $E^{>k} G \phi$ is actually counting children paths where $\phi$ is true in each node of the paths. Since each node can have one parent only, then each path in $T$ can be distinguished by the leaf nodes. We can count leaf nodes, and hence paths, with formula $(\neg\langle\downarrow\rangle T)>k$. Paths starting at a node $o$ where $\phi$ is true at each node can be denoted by $\mu x . F(\phi) \wedge[\langle\uparrow\rangle x \vee(o \wedge o=1)]$. It is now easy to see that $T$ satisfies 
$F\left(E^{>k} G \phi\right)$, if and only if, there are at least $k$ children paths starting at node $o$, such that $\phi$ holds at each node of the paths.

The remaining case is analogous.

Regarding the size of translation, it is clear that $F$ does not introduce duplications, and the proof also goes straightforward by induction on the structure of the input formula.

In order to show that $\mu$ TLIN is at least exponentially more succinct than the graded $\mu$-calculus, we then first define the logic.

Definition 5.6 (Graded $\mu$-calculus). The set of formulas of the graded $\mu$-calculus is defined by the following grammar.

$$
\phi:=p|x| \neg \phi|\phi \vee \phi|\langle m\rangle \phi|\mu x . \phi| E^{>k} X \phi
$$

Modalities does not include two-way navigation, that is, $m \in\{\downarrow, \rightarrow\}$. Formulas are interpreted as node subsets of a given tree structure. The interpretation in the formula fragment corresponding to $\mu$ TLIN is the same as in $\mu$ TLIN. The formula $E^{>k} X \phi$ is interpreted as in GCTL.

We now recall a Theorem from Bianco et al. regarding the exponential succinctness of GCTL with respect to the graded $\mu$-calculus.

Theorem 5.7 (GCTL succinctness [BMM10, BMM12]). There is a GCTL formula $\phi$, such that every equivalent graded $\mu$-calculus formula has exponential size with respect to $\phi$.

From Theorems 5 and 5.7, it is then easy to infer an exponential succinctness of $\mu$ TLIN formulas with respect to the graded $\mu$-calculus.

Corollary 5.8 ( $\mu$ TLIM succinctness). For any tree $T$ and valuation $V$, there is a $\mu$ TLIN formula $\phi$, such that every graded $\mu$-calculus formula $\psi$ is that if

$$
\llbracket \phi \rrbracket_{V}^{T} \neq \varnothing \text { if and only if } \llbracket \psi \rrbracket_{V}^{T} \neq \varnothing,
$$

then $\psi$ has exponential size with respect to $\phi$.

\section{Decidability}

In this Section, we show that the $\mu$ TLIN is decidable. This is achieved by a reduction to the two-way $\mu$-calculus Var98]. Before describing the reduction, we first need to recall a well-known bijection between binary and $n$-ary trees.

6.1. Binary trees. There is well-known bijection between $n$-ary unranked trees and binary unranked trees [HVP05]. One of the edges in the binary trees represents the first child relation, whereas the other edge represent the following sibling relation. In Figure 2 there is a graphical representation of this bijection. Therefore, from now on, without loss of generality, we will consider binary trees only.

At the logic level, we now reinterpret the modal formula $\langle m\rangle \phi$ as follows:

- formula $\langle\downarrow\rangle \phi$ selects the nodes where $\phi$ holds in its first child;

- formula $\langle\uparrow\rangle \phi$ selects the nodes whose parent satisfy $\phi$;

- $\langle\rightarrow\rangle \phi$ holds in nodes where $\phi$ is satisfied by its following sibling; and

- $\langle\leftarrow\rangle \phi$ satisfies nodes such that $\phi$ holds in its previous sibling. 

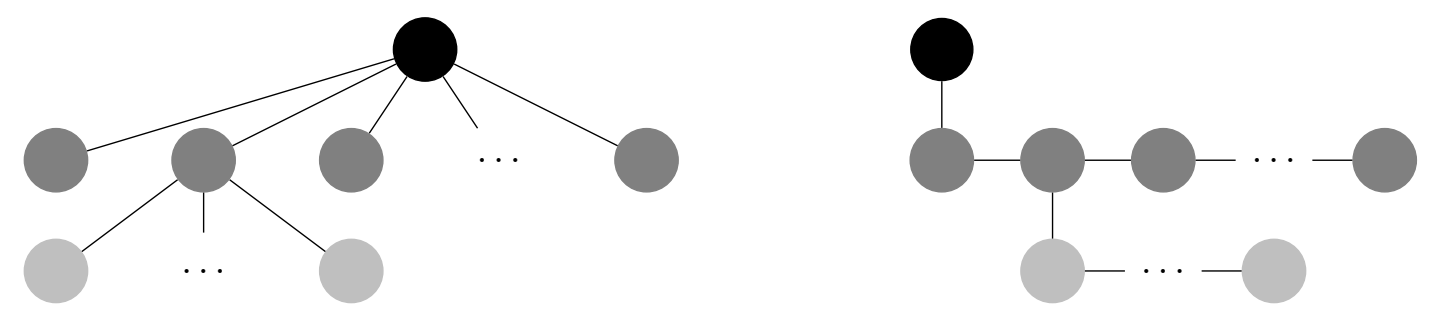

Figure 2: Example of the bijection between $n$-ary and binary trees.

Proposition 6.1. Consider a bijection $f$ from n-ary trees to binary trees, as the one in HVP05. We have the following:

- for any $n$-ary tree $T$, valuation $V$, and $\mu T L I N$ formula $\phi$, there is a $\mu T L I N$ formula $\psi$ such that

$$
\llbracket \phi \rrbracket_{V}^{T}=\llbracket \psi \rrbracket_{V}^{f(T)}
$$

- and for any binary tree $B$, valuation $V$, and $\mu T L I N$ formula $\psi$, there is a $\mu T L I N$ formula $\phi$ such that

$$
\llbracket \psi \rrbracket_{V}^{B}=\llbracket \phi \rrbracket_{V}^{f^{-1}(B)} .
$$

Proof. Consider the first item. We proceed by induction on the structure of $\phi$. The base and most inductive cases are immediate. We consider the modal case only. If the input formula has the form $\langle\downarrow\rangle \varphi$, then $\psi$ is $\langle\downarrow\rangle \mu x . \varphi^{\prime} \vee\langle\rightarrow\rangle x$, where $\varphi^{\prime}$ is the equivalence (by induction) of $\varphi$. When the input formula is $\langle\uparrow\rangle \varphi$, then $\psi$ is $\mu x .\langle\uparrow\rangle \varphi^{\prime} \vee\langle\leftarrow\rangle x$. The cases for $\langle\rightarrow\rangle \varphi$ and $\langle\leftarrow\rangle \varphi$ are analogous. The second item is trivial: $\psi$ is defined as $\phi$.

6.2. Reduction. We now provide a reduction from $\mu$ TLIN to the two-way $\mu$-calculus, that is, we will describe an encoding of counting formulas $\phi>k$ into plain $\mu$-calculus formulas. For this purpose, we first define a $\mu$-calculus formula counting from the root.

Definition 6.2. We define the following formulas for $i>1$ :

$$
\begin{aligned}
C_{0}^{\phi}= & \mu x . \phi \vee\langle\downarrow\rangle x \vee\langle\rightarrow\rangle x \\
C_{1}^{\phi}= & \mu x .\left(\phi \wedge\left(\langle\downarrow\rangle C_{0}^{\phi} \vee\langle\rightarrow\rangle C_{0}^{\phi}\right)\right) \vee\left(\neg \phi \wedge\langle\downarrow\rangle C_{0}^{\phi} \wedge\langle\rightarrow\rangle C_{0}^{\phi}\right) \vee\langle\downarrow\rangle x \vee\langle\rightarrow\rangle x \\
C_{i}^{\phi}= & \mu x .\left(\phi \wedge\left(\langle\downarrow\rangle C_{i-1}^{\phi} \vee\langle\rightarrow\rangle C_{i-1}^{\phi} \vee \bigvee_{k_{1}+k_{2}=i-2}\langle\downarrow\rangle C_{k_{1}}^{\phi} \wedge\langle\rightarrow\rangle C_{k_{2}}^{\phi}\right)\right) \vee \\
& \left(\neg \phi \wedge \bigvee_{k_{1}+k_{2}=i-1}\langle\downarrow\rangle C_{k_{1}}^{\phi} \wedge\langle\rightarrow\rangle C_{k_{2}}^{\phi}\right) \vee\langle\downarrow\rangle x \vee\langle\rightarrow\rangle x
\end{aligned}
$$

From the root node, $C_{k}^{\phi}$ counts at least $k+1$ nodes satisfying $\phi$. In Figure 3 there is an example model for $C_{3}^{p_{1}}$ holding at the root. $C_{3}^{p_{1}}$ counts at least 4 nodes named $p_{1}$.

Recall that, in a tree, the root is the only node without a parent, hence the root $r$ can be denoted by the formula $\neg\langle\uparrow\rangle \top \wedge \neg\langle\leftarrow\rangle T$. We can thus reach the root from any other node with the following formula:

$$
\mu x . r \vee\langle\uparrow\rangle x \vee\langle\leftarrow\rangle x
$$




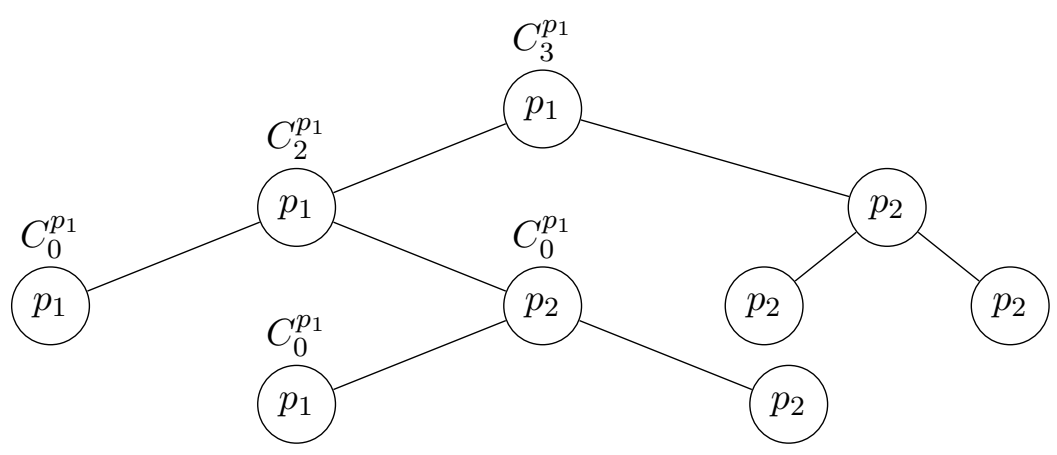

Figure 3: Example model for $C_{3}^{p_{1}}$ holding at the root

Now, with the help of $C_{k}^{\phi}$, we can now show how to encode counting formulas into the simple $\mu$-calculus (without counting constructs).

Lemma 1. For any tree $T$ and valuation $V$, we have the following:

$$
\llbracket \phi>k \rrbracket_{V}^{T}=\llbracket \mu x .\left(C_{k}^{\phi} \wedge r\right) \vee\langle\uparrow\rangle x \vee\langle\leftarrow\rangle x \rrbracket_{V}^{T}
$$

Proof. The proof goes by induction on $k$ in $C_{k}^{\phi}$. The base cases $C_{0}^{\phi}$ and $C_{1}^{\phi}$ are trivial. For the induction step we distinguish two cases:

- Assume $\phi$ holds at the root, we have then 1 occurrence of $\phi$. It is then easy to see by induction that

$$
\langle\downarrow\rangle C_{k-1}^{\phi} \vee\langle\rightarrow\rangle C_{k-1}^{\phi} \vee \bigvee_{k_{1}+k_{2}=k-2}\langle\downarrow\rangle C_{k_{1}}^{\phi} \wedge\langle\rightarrow\rangle C_{k_{2}}^{\phi}
$$

counts $k$ occurrences of $\phi$. There are then $k+1$ occurrence of $\phi$.

- Assume $\phi$ does not hold at the root. Then there are two subcases:

- There are occurrences of $\phi$ in both subtrees, in which cases by induction we know that

$$
\bigvee_{k_{1}+k_{2}=k-1}\langle\downarrow\rangle C_{k_{1}}^{\phi} \wedge\langle\rightarrow\rangle C_{k_{2}}^{\phi}
$$

counts $k+1$ occurrence of $\phi$.

- The other case is when there are not occurrences of $\phi$ in one of the subtrees. We then apply recursion on the subtrees $(\langle\downarrow\rangle x \vee\langle\rightarrow\rangle x)$. The rest of the proof is immediate by induction on the height of the tree model.

Now that we can encode the counting formulas into plain two-way $\mu$-calculus, then we can infer that $\mu$ TLIN is decidable due to the fact that $\mu$-calculus is decidable. However, the encoding of counting formulas results in exponentially larger $\mu$-calculus formulas.

Theorem 6.3. $\mu$ TLIN is decidable in double exponential time.

Proof. Observe in Definition 6.2 that $C_{k}^{\phi}$ encodes numerical constraints by nesting $k$ modalities on $\phi$. That is, $\langle m\rangle C_{k-1}^{\phi},\langle m\rangle\langle m\rangle C_{k-2}^{\phi}, \ldots,\langle m\rangle \ldots\langle m\rangle C_{0}^{\phi}$ are all subformulas of $C_{k}^{\phi}$. Since $k$ is in binary form, this implies that there are $2^{k}$ different occurrences of $\phi$ in $C_{k}^{\phi}$. That is, the size of $C_{k}^{\phi}$ is exponentially greater than the sum of the sizes of $\phi$ and $k$. Now, by the fact that the $\mu$-calculus is EXPTIME-complete [BLMV06], and by Lemma 1, we conclude the doubly exponential time complexity bound. 
The graded $\mu$-calculus KSV02 was also shown to be decidable by a reduction to the plain two-way $\mu$-calulculus by Bárcenas in Bar11, then the expressive power of $\mu$ TLIN, the graded $\mu$-calculus and the plain two-way $\mu$-calculus all coincide.

Being $\mu$ TLIN decidable, and by Theorems 3.8 and 4.5, we can then use as a reasoning framework for XPath queries with schema and counting constraints. However, the complexity bound for decidability can be improved. In the rest of the paper, we will describe a satisfiability algorithm with single exponential time complexity. Before defining the algorithm, we first describe a Fischer-Ladner representation of tree models.

\section{Fischer-LADNER TREeS}

This is a section of preliminaries for the satisfiability algorithm. It is described a syntactic representation of tree models.

For the algorithm, we consider formulas in negation normal form (NNF) only.

Definition 7.1 (Negation Normal Form). In the negation normal form $\operatorname{nnf}(\phi)$ of a formula $\phi$, negation occurs only immediately above of propositions, $T$ and modal subformulas $\langle m\rangle T$. This is obtained by the following rules together with the usual DeMorgan's:

$$
\begin{aligned}
\neg\langle m\rangle \phi & =\langle m\rangle \neg \phi \vee \neg\langle m\rangle \top, & \neg(\phi>k) & =\phi \leqslant k, \\
\neg(\phi \leqslant k) & =\phi>k, & \neg \mu x . \phi & =\mu x . \neg \phi\left[{ }^{x} / \neg x\right] .
\end{aligned}
$$

Note that, for technical convenience, we consider an extension of formulas. This extension consists of less than counting formulas $\phi \leqslant k$ and the true formula $T$ with the obvious semantics.

We require some notation before defining the Fischer-Ladner closure.

Since integers associated to counting constraints are assumed to be in binary form, we thus define counter formulas as a boolean combination of propositions denoting an integer number. For example, for a sequence of propositions $p_{1}, p_{2}, \ldots$, the integer 1 is written $p_{1} \wedge \bigwedge_{i>1} \neg p_{i}$, and the integer 5 (101 in binary) is written $p_{3} \wedge \neg p_{2} \wedge p_{1} \wedge \bigwedge_{i>4} \neg p_{i}$. The amount of propositions required to define the counters of formula $\phi$ is bounded by $\max K(\phi)$.

Definition 7.2. We define $\max K(\phi)$ as follows:

$$
\begin{aligned}
& \max K(p)=\max K(x)=\max K(\top)=0 \\
& \max K(\langle m\rangle \phi)=\max K(\neg \phi)=\max K(\mu x . \phi)=\max K(\phi) \\
& \max K\left(\phi_{1} \vee \phi_{2}\right)=\max K\left(\phi_{1} \wedge \phi_{2}\right)=\max K\left(\phi_{1}\right)+\max K\left(\phi_{2}\right) \\
& \max K(\phi \# k)=\max K(\phi)+(k+1)
\end{aligned}
$$

When clear from the context, we often simply write $\max K$.

Definitions of counters and flags is now given.

Definition 7.3 (Counters and flags). For a counting subformula $\phi \# k$ of a given formula:

- a counter $\phi^{k^{\prime}}$ set to $k^{\prime}$ is a sequence of fresh propositions occurring positively in the binary coding of the integer $k^{\prime}$; and

- a flag $\phi^{\# k}$ is a fresh proposition.

For instance, for the integer 5 coded as $c_{2} \wedge \neg c_{1} \wedge c_{0}$, we write $\phi^{5}$ to denote $c_{2}, c_{0}$, where $c_{i}$ are the corresponding propositions for the counting formula $\phi \# k$. 
The Fischer-Ladner closure of a given formula is the set of its subformulas together with their negation normal form, such that the fixed-points are expanded once. Additionally, a counter and a flag for each counting subformula are also considered in the closure. All these information is obtained with the help of the relation $R^{F L}$.

Definition 7.4. We define the following binary relation $R^{F L}$ over formulas for $i=1,2$ :

$$
\begin{array}{lrr}
R^{F L}(\phi, \operatorname{nnf}(\phi)) & R^{F L}\left(\phi_{1} \wedge \phi_{2}, \phi_{i}\right) & R^{F L}\left(\phi_{1} \vee \phi_{2}, \phi_{i}\right) \\
R^{F L}(\langle m\rangle \phi, \phi) & R^{F L}(\mu x . \phi, \phi[\mu x . \phi / x]) & R^{F L}(\phi \# k, \phi) \\
R^{F L}\left(\phi \# k, \phi^{\max K}\right) & R^{F L}\left(\phi \# k, \phi^{\# k}\right) & R^{F L}(\phi \# k, \psi)
\end{array}
$$

where $\psi=\mu x_{1} .\left(\mu x_{2} . \phi \vee\langle\downarrow\rangle x_{2} \vee\langle\rightarrow\rangle x_{2}\right) \vee\langle\uparrow\rangle x_{1} \vee\langle\leftarrow\rangle x_{1}$. Notice that if $\phi$ is true in a model, then $\psi$ is true in every node of the model. We use $\psi$ to provide the necessary information for $\phi$ to navigate through the entire model.

We are now ready to define the Fischer-Ladner closure.

Definition 7.5 (Fischer-Ladner Closure). The Fischer-Ladner closure of a given formula $\phi$ is defined as $\operatorname{FL}(\phi)=\mathrm{FL}(\phi)_{k}$, such that $k$ is the smallest integer satisfying $\operatorname{FL}(\phi)_{k+1}=$ $\operatorname{FL}(\phi)_{k}$, where:

$$
\begin{aligned}
& \mathrm{FL}(\phi)_{0}=\{\phi\} \\
& \operatorname{FL}(\phi)_{i+1}=\mathrm{FL}(\phi)_{i} \cup\left\{\psi^{\prime} \mid R^{F L}\left(\psi, \psi^{\prime}\right), \psi \in \mathrm{FL}(\phi)_{i}\right\}
\end{aligned}
$$

The lean set of a given formula contains propositions, modal and counting subformulas, together with counters and flags.

Definition 7.6 (Lean). Given a formula $\phi$ and a proposition $p^{\prime}$ not occurring in $\phi$, we define the lean as follows for all $m \in M$ :

$$
\operatorname{lean}(\phi)=\left\{p,\langle m\rangle \psi, \psi \# k, \psi^{\max K}, \psi^{\# k} \in \mathrm{FL}(\phi)\right\} \cup\left\{\langle m\rangle T, p^{\prime}\right\}
$$

The lean set contains all the required information to define tree nodes: propositions serve as labels, modal subformulas define the topology of the tree, and counters and flags serve to verify counting subformulas.

As in BGLS11, CGLV10, GLS07, the single exponential time complexity of the satisfiability algorithm mainly relies in the size of the lean set (tree nodes are defined as subsets of the lean). Since counters are coded in binary, it is then easy to see that the size of the lean set is not significantly increased with respect to the original formula.

Lemma 2. The cardinality of lean $(\phi)$ is linear with respect to the size of $\phi$.

Proof. The proof goes by structural induction on $\phi$.

We consider only the case for counting subformuals $R^{F L}\left(\phi^{\prime} \# k, \psi\right)$. Now recall that a counter is defined in terms of a boolean combination of propositions, that is, for each counting subformula $\phi^{\prime} \# k$ only $\log (\max K)(\max K$ in binary) new propositions are introduced in the lean. Since the size of $\phi^{\prime} \# k$ is defined by $\left|\phi^{\prime}\right|+\log (k+1)$, and by the definition of $\max K$, the counters then produce no increment in the size of the lean. 
Example 7.7. Consider the following formulas for $m \in\{\downarrow, \rightarrow, \uparrow, \leftarrow\}$ :

$$
\begin{aligned}
\phi & =\left[\left(p_{1}>1\right) \wedge p_{2}\right]>4 & \psi & =\left(p_{1}>1\right) \wedge p_{2} \\
\phi_{0} & =\mu x . \psi \vee \bigvee_{\forall m}\langle m\rangle x & \psi_{0} & =\mu x . p_{1} \vee \bigvee_{m}\langle m\rangle x
\end{aligned}
$$

The lean of $\phi$ is thus defined as follows for $m \in\{\downarrow, \rightarrow, \uparrow, \leftarrow\}$ :

$$
\operatorname{lean}(\phi)=\left\{p_{1}, p_{2}, \phi, p_{1}>1, \psi^{7}, p_{1}^{7}, \psi^{>4}, p_{1}^{>1},\langle m\rangle \phi_{0},\langle m\rangle \psi_{0}, p^{\prime},\langle m\rangle \top\right\}
$$

$\max K=7$. Now recall that $\phi^{7}$ denote 3 propositions that serve to express the binary coding of the integers from 0 to 7 .

We are now ready to define the syntactic notion of tree nodes.

Definition 7.8 ( $\phi$-Nodes). Given a formula $\phi$, a $\phi$-node $n^{\phi}$ is defined as a subset of lean $(\phi)$, such that:

- at least one proposition of $\phi$ occurs;

- if $\langle m\rangle \psi$ occurs, then $\langle m\rangle\rceil$ also does;

- both $\langle\leftarrow\rangle T$ and $\langle\uparrow\rangle T$ can not occur;

- counting formulas are always present;

- exactly one counter for each counting formula is present, i.e., if $\phi \# k \in n^{\phi}$, then $\phi^{k^{\prime}} \in n^{\phi}$;

- counters must be consistent with counting formulas and flags, i.e., $\psi^{k_{0}}, \psi \leqslant k \in n$, if and only if, $k_{0} \leqslant k$, and $\psi^{k_{0}}, \psi^{>k} \in n$, if and only if, $k_{0}>k$.

The set of $\phi$-nodes is written $N^{\phi}$. If the context is clear, we often call a $\phi$-node simply a node, and we write $n$ instead of $n^{\phi}$.

We now define trees as triples $\left(n, X_{1}, X_{2}\right)$, where $n$ is the root of the tree and $X_{1}$ and $X_{2}$ are the respective left and right subtrees.

Definition 7.9 (Fischer-Ladner trees). Given a formula, a Fischer-Ladner tree, or simply a tree, is inductively defined as follows:

- the empty set $\varnothing$ is a tree;

- the triple $\left(n^{\phi}, X_{1}, X_{2}\right)$ is also a tree, provided that $X_{1}$ and $X_{2}$ are also trees.

Example 7.10. Consider $\phi, \psi, \phi_{0}, \psi_{0}$ from Example 7.7. We define the following syntactic tree model for $\phi$ :

$$
T=\left(n_{0},\left(n_{1},\left(n_{3}, \varnothing, \varnothing\right),\left(n_{4}, \varnothing, \varnothing\right)\right),\left(n_{2},\left(n_{5}, \varnothing, \varnothing\right),\left(n_{6}, \varnothing, \varnothing\right)\right)\right.
$$

where

$$
\begin{aligned}
& n_{0}=\left\{p_{2}, \phi, p_{1}>1, p_{1}^{2}, p_{1}^{>1}, \psi^{5}, \psi^{>4},\langle\downarrow\rangle \psi_{0},\langle\rightarrow\rangle \psi_{0},\langle\downarrow\rangle \phi_{0},\langle\rightarrow\rangle \phi_{0},\langle\downarrow\rangle T,\langle\rightarrow\rangle \top\right\} \\
& n_{1}=\left\{p_{2}, \phi, p_{1}>1, p_{1}^{2}, p_{1}^{>1}, \psi^{1},\langle\downarrow\rangle \psi_{0},\langle\rightarrow\rangle \psi_{0},\langle\uparrow\rangle \psi_{0},\langle\downarrow\rangle \phi_{0},\langle\rightarrow\rangle \phi_{0},\langle\uparrow\rangle \phi_{0},\langle\downarrow\rangle \top,\langle\rightarrow\rangle \top,\langle\uparrow\rangle \top\right\} \\
& n_{2}=\left\{p_{2}, \phi, p_{1}>1, p_{1}^{2}, p_{1}^{>1}, \psi^{3},\langle\downarrow\rangle \psi_{0},\langle\rightarrow\rangle \psi_{0},\langle\leftarrow\rangle \psi_{0},\langle\downarrow\rangle \phi_{0},\langle\rightarrow\rangle \phi_{0},\langle\leftarrow\rangle \phi_{0},\langle\downarrow\rangle \top,\langle\rightarrow\rangle \top,\langle\leftarrow\rangle \top\right\} \\
& n_{3}=\left\{p_{1}, \phi, p_{1}>1, p_{1}^{1},\langle\uparrow\rangle \phi_{0},\langle\uparrow\rangle \psi_{0},\langle\uparrow\rangle \top\right\} \\
& n_{4}=\left\{p_{1}, \phi, p_{1}>1, p_{1}^{1},\langle\leftarrow\rangle \phi_{0},\langle\leftarrow\rangle \psi_{0},\langle\leftarrow\rangle \top\right\} \\
& n_{5}=\left\{p_{2}, \phi, p_{1}>1, \psi^{1},\langle\uparrow\rangle \phi_{0},\langle\uparrow\rangle \psi_{0},\langle\uparrow\rangle \top\right\} \\
& n_{6}=\left\{p_{2}, \phi, p_{1}>1, \psi^{1},\langle\leftarrow\rangle \phi_{0},\langle\leftarrow\rangle \psi_{0},\langle\leftarrow\rangle \top\right\}
\end{aligned}
$$

Figure 4 depicts a graphical representation of $T$. 


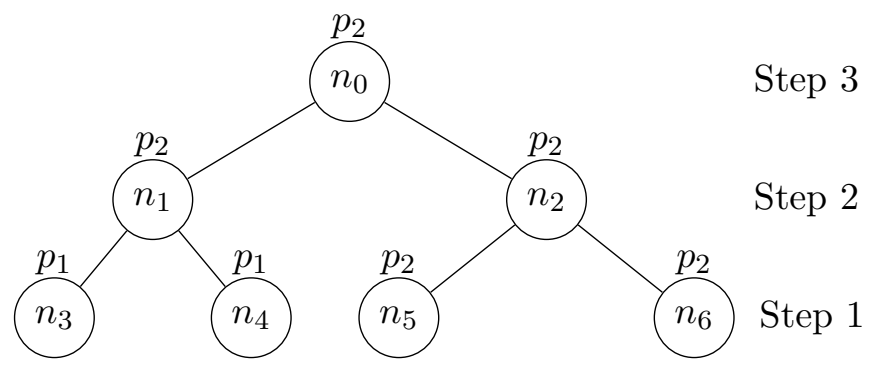

Figure 4: Fischer-Ladner tree model for $\phi=\left[\left(p_{1}>1\right) \wedge p_{2}\right]>4$

\section{SATisfiability}

In this Section, we introduce a satisfiability algorithm for $\mu$ TLIN in the style of FischerLadner [BGLS11, DL10]. Tree nodes are defined from an extension of the classical FischerLadner closure. The extension consists of counters (boolean combination of fresh propositions encoding integer values in binary) that are used to verify counting formulas. Tree models are built in a bottom-up manner, that is, starting from the leaves, parent nodes are consistently added until a witness tree for the formula in question is found. At each step in this process, counters must be consistent with the counters of children nodes and the formulas that hold in the current parent node.

8.1. The algorithm. The satisfiability algorithm, described in Algorithm 1, builds candidate trees in a bottom-up manner: iteratively, starting from leaf nodes, we check at each step if the input formula is satisfied by candidate trees, in case the formula is not satisfied, we consistently add parents to previously built trees. The algorithm returns 1 if a satisfying tree is found. In case a satisfying tree could not be found, and no more candidate trees can be built, then the algorithm returns 0 .

Example 8.1. Consider the formula $\phi$ defined in Example 7.7. Then the Fischer-Ladner tree defined in Example 7.10 is built by the algorithm in 3 steps. In the first step, all the leaves are considered, that is, nodes without children, such that the counters are properly initialized (Definition 8.3). It is then easy to see that $n_{3}, n_{4}, n_{5}, n_{6}$ are all leaves. Since $p_{1}$ is occurring in both, $n_{3}$ and $n_{4}$, then the counter $p_{1}^{1}$ is also in the same nodes. Since both $p_{2}$ and $p_{1}>1$ are in $n_{5}$ and $n_{6}$, then $\psi=p_{2} \wedge p_{1}>1$ is true in both nodes, and consequently $\psi^{1}$ is also in $n_{5}$ and $n_{6}$. However, none of the leaves satisfies $\phi$, then, in the second step, $n_{1}$ is added as parent to both $n_{3}$ and $n_{4} . n_{2}$ is also added as parent to $n_{5}$ and $n_{6}$. Since $\psi$ is true in $n_{1}$ and $n_{2}$, then the counter for $\psi$ is incremented in both nodes. Resulting that in $n_{1}$ we have $\psi^{1}$, and in $n_{2}$ we have $\psi^{3}$. However, none of the trees built in step 2 satisfies $\phi$. In step $3, n_{0}$ is then added as parent of $n_{1}$ and $n_{2}$. Since $\psi$ holds in $n_{0}$, then we update the counter to $\psi^{5}$, and $\phi$ is then finally satisfied. This process is depicted in Figure 4 ,

We now provide a precise description of the algorithm components.

If a tree $T$ is a model for a formula $\phi$, it is said that $T$ satisfies (entails) $\phi$. We now give a precise definition of this entailment relation. 


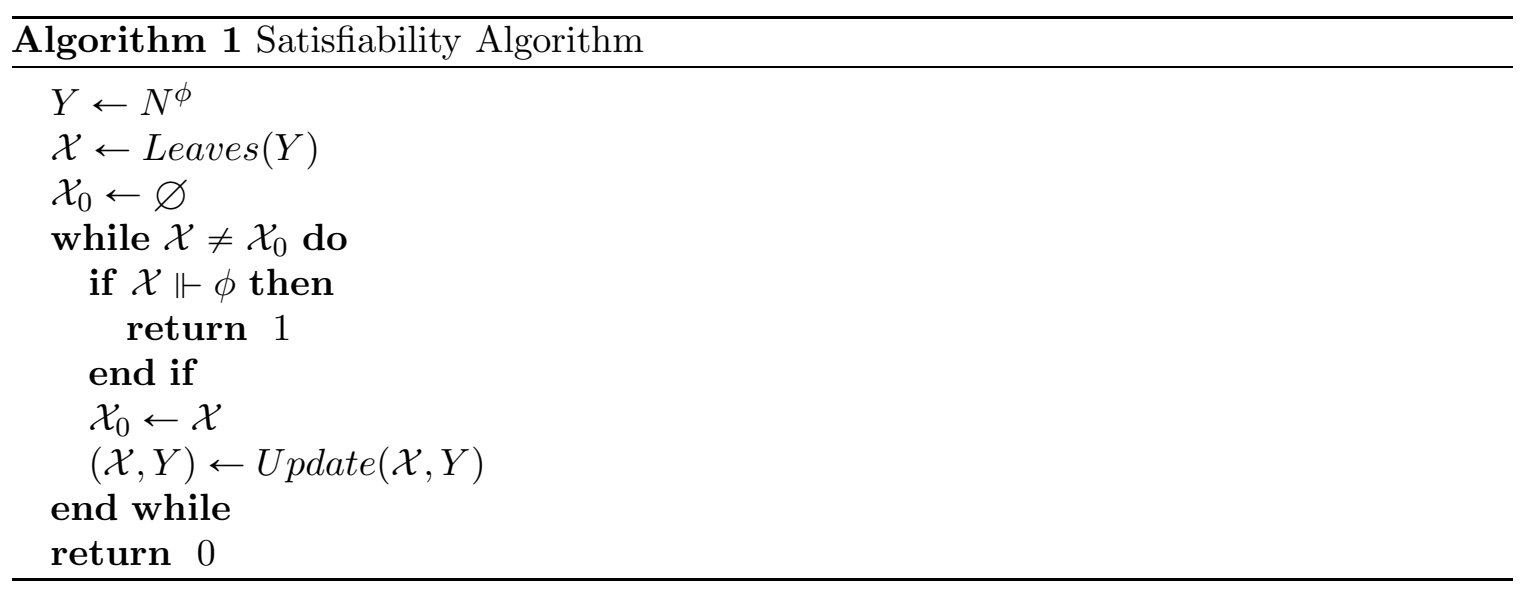

Definition 8.2. The entailment of a formula by a node is defined by:

$$
\begin{array}{llll}
\overline{n \vdash \top} & \frac{\phi \in n}{n \vdash \phi} & \frac{\phi \notin n}{n \vdash \neg \phi} & \frac{n \vdash \phi \quad n \vdash \psi}{n \vdash \phi \wedge \psi} \\
\frac{n \vdash \phi}{n \vdash \phi \vee \psi} & \frac{n \vdash \psi}{n \vdash \phi \vee \psi} & \frac{n \vdash \phi[\mu x . \phi / x]}{n \vdash \mu x . \phi} &
\end{array}
$$

The entailment relation is now extended for trees and formulas. A formula $\phi$ is satisfied by a tree $X$, written $X \Vdash \phi$, if and only if,

- there is a node $n$ in $X$, such that $n \vdash \phi$;

- formulas of the forms $\langle\uparrow\rangle \psi$ and $\langle\leftarrow\rangle \psi$ do not occur in the root of $X$; and

- all the flags are in the root.

A set of trees $\mathcal{X}$ entails a formula $\phi$, written $\mathcal{X} \Vdash \phi$, if and only if, there is a tree $X$ in $\mathcal{X}$ s.t. $X \Vdash \phi$.

The relation $\forall$ is defined as expected.

The sef of leaves contains nodes without children. In the leaves, counters are also properly initialized.

Definition 8.3 (Leaves). Given set of nodes $X$, the set of leaves is defined as follows:

$$
\text { Leaves }(X)=\left\{(n, \varnothing, \varnothing) \mid n \in X,\langle\downarrow\rangle \phi,\langle\rightarrow\rangle \phi \notin n,\left[\left(\phi^{1} \in n, n \vdash \phi\right) \text { or }\left(\phi^{0} \in n, n \nvdash \phi\right)\right]\right\}
$$

Recall that counting formulas are true in the entire model when satified, then counting formulas are always present in every $\phi$-node. The corresponding counters will be updated each time they find a witness. Notice that counting subformulas with the form $\psi>k$ may not be true at earlier steps of the algorithm. We then use flags to identify when those formulas become true, that is, when we find more than $k$ witnesses of $\psi$, we then turn on the flag $\psi^{>k}$. Once a flag is turned on, it is copied to parents at each further step. It is then required to have all the flags in the root in order to ensure that counting subformulas $\psi>k$ are all satisfied.

For the step case in the algorithm, if newly built trees do not satisfy the formula, then new candidate trees are constructed by adding a parent to previously built trees. This is done by the Update function, which is defined with the help of the following auxiliary functions. 
A node $n$ containing a modal formula $\langle m\rangle \psi$ can be linked to another node $n^{\prime}$ through a modality $m$, if and only if, there is a witness of $\psi$ in $n^{\prime}$, that is, $n^{\prime} \vdash \psi$. This notion is defined by the relation $\Delta_{m}$.

Definition 8.4. Given two nodes $n_{1}, n_{2}$ and formula $\phi$, we say that the nodes are modally consistent with respect to the formula $\Delta_{m}\left(n_{1}, n_{2}\right)$ for $m \in\{\downarrow, \rightarrow\}$, if and only if, for all formulas $\langle m\rangle \psi_{1},\langle\bar{m}\rangle \psi_{2} \in \operatorname{lean}(\phi)$, we have that:

- $\langle m\rangle \psi_{1} \in n_{1}$ if and only if $n_{2} \vdash \psi_{1}$, and

- $\langle\bar{m}\rangle \psi_{2} \in n_{2}$ if and only if $n_{1} \vdash \psi_{2}$.

Example 8.5. Consider the algorithm execution described in Example 8.1. In the second step, when linking $n_{1}$ with $n_{3}$ and $n_{4}$, note that $\Delta_{\downarrow}\left(n_{1}, n_{3}\right)$ and $\Delta_{\rightarrow}\left(n_{1}, n_{4}\right)$. This is because $\phi_{0}$ and $\psi_{0}$ are both true in $n_{3}$ and $n_{4}$, that is, $n_{3} \vdash \phi_{0}, n_{3} \vdash \psi_{0}, n_{4} \vdash \phi_{0}$, and $n_{4} \vdash \psi_{0}$.

When adding parents, it is also necessary to ensure that counting formulas are satisfied. Recall that, according to the definition of $\phi$-nodes, counting formulas and flags are consistent with counters. It is then only required to update the counters and to copy the flags that are already in the subtrees. We have two cases. The first one is when we add a parent to both, a left and a right subtrees. The second case is when a parent is added to one subtree only. Consider the first case.

Definition 8.6. It is said that three nodes $n_{0}, n_{1}, n_{2}$ are consistent with respect to their counters, denoted by \# $\left(n_{0}, n_{1}, n_{2}\right)$, if and only if,

- $\psi^{k_{0}} \in n_{0}$ and $n_{0} \vdash \psi$, if and only if, $\psi^{k_{1}} \in n_{1}, \psi^{k_{2}} \in n_{2}$ and $k_{0}=k_{1}+k_{2}+1$ if $k_{0} \leqslant \max K$, otherwise $k_{0}=\max K$

- $\psi^{k_{0}} \in n_{0}$ and $n_{0} \nvdash \psi$, if and only if, $\psi^{k_{1}} \in n_{1}, \psi^{k_{2}} \in n_{2}$ and $k_{0}=k_{1}+k_{2}$ if $k_{0} \leqslant \max K$, otherwise $k_{0}=\max K$; and

- if $\psi^{>k} \in n_{i}$ for any $i \in\{1,2\}$, then $\psi^{>k} \in n_{0}$.

The second case $\left(\#\left(n_{0}, n_{i}\right)\right)$ is defined in an analogous manner.

Example 8.7. Consider again the execution described in Example 8.1. Since $\psi^{1} \in n_{1}$, $\psi^{3} \in n_{2}$ and $n_{0} \vdash \psi$, it is then consistent that $\psi^{5} \in n_{0}$, and hence \# $\left(n_{0}, n_{1}, n_{2}\right)$.

Recall that the Update function is used to consistently add parents to previously built trees. Now, with the notions of modal and counter consistency (Definitions 8.4 and 8.6) already defined, we are now ready to give a precise description of the Update function.

Definition 8.8. Given a set of trees $\mathcal{X}$ and a set of nodes $Y$, the function $\operatorname{Update}(\mathcal{X}, Y)$ is defined as the tuple $\left(\mathcal{X}^{\prime}, Y^{\prime}\right)$, such that:

- $\mathcal{X}^{\prime}=\left\{\left(n, X_{\downarrow}, X_{\rightarrow}\right) \mid n \in Y, X_{i} \in \mathcal{X}, \Delta_{i}\left(n, n_{i}\right), \#\left(n, n_{1}, n_{2}\right)\right\}$, where $i=\downarrow, \rightarrow$ and $n_{i}$ is the root of $X_{i}$; or

- $\mathcal{X}^{\prime}=\left\{\left(n, X_{\downarrow}, X_{\rightarrow}\right) \mid n \in Y, X_{i} \in \mathcal{X}, \Delta_{i}\left(n, n_{i}\right), \#\left(n, n_{i}\right)\right\}$ in case $X_{j}=\varnothing$ with $i \neq j$; and

- $Y^{\prime}=Y \backslash\{n\}$.

We now prove that the algorithm is correct. We also describe a single exponential bound in the time complexity of the algorithm. 
8.2. Correctness and Complexity. It is easy to see that the algorithm has a finite number of steps if we notice that the number of nodes is finite and that the Update function is monotone.

In order to show that the algorithm is correct, we then prove it to be sound and complete.

Theorem 8.9 (Soundness). If the algorithm returns 1 for the input formula $\phi$, then there is tree model satisfying $\phi$.

Proof. By assumption, there is a triple $X$ such that $X \Vdash \phi$. We will now construct a tree model $T$ from $X$.

- The set of propositions $\mathcal{P}$ are the ones in lean $(\phi)$.

- The nodes of $T$ are $\mathcal{N}^{\phi}$.

- We now define the edges of $T$. For every triple $\left(n, X_{1}, X_{2}\right)$ of $X$, we define $\mathcal{R}(n, \downarrow)=n_{1}$ and $\mathcal{R}(n, \rightarrow)=n_{2}$, provided that $n_{1}$ and $n_{2}$ are the respective roots of $X_{1}$ and $X_{2}$.

- We label the nodes in the obvious manner: if $p \in n$, then $p \in \mathcal{L}(n)$.

It is now shown by structural induction on $\phi$ that $T$ satisfies $\phi$. All cases are straightforward. For the case of fixed-point subformulas, recall that there is an equivalent finite unfolding, that is: $\mu x \cdot \psi \equiv \phi[\mu x \cdot \phi / x]$ [BLMV06, BGLS11].

For completeness it is assumed that there is a satisfying tree $T$ for the formula $\phi$, and then it is shown that the algorithm returns 1. The proof comes in two steps: we first construct an equivalent lean labeled version of $T$, and then we show that the algorithm can actually construct such lean labeled tree.

Definition 8.10. Given a satisfying tree $T$ of a formula $\phi$, we define its lean version $X^{T}$ as follows:

- $X^{T}$ has the same nodes and shape than $T$;

- each node $n$ in $X^{T}$ is labeled with the formulas $\psi$ in lean $(\phi)$ such that

$-n$ in $T$ satisfies $\psi$, and

- the labels corresponding to the counters are pinned up in a similar manner as the algorithm does, that is, in an increasing order (with bound maxK) from bottom-up in the tree.

Lemma 3. If a tree $T$ satisfies a formula $\phi$, then $\phi$ is entailed by $X^{T}$.

Proof. We proceed by induction on the derivation of $n \vdash \phi$. Most cases are immediate by induction and the construction of $X^{T}$.

For the fixpoint case $\mu x . \psi$, we test $\psi[\mu x . \psi / x]$. We then proceed by structural induction again. This is also straightforward since variables, and hence unfolded fixed-points, can only occur in the scope of a modality or a counting formula.

One crucial point in the completeness proof is to show that $N^{\phi}$ contains enough nodes to satisfy $\phi$. It is well-known that the standard Fischer-Ladner construction of models provides the required amount of nodes for simple $\mu$-calculus formulas without counting BLMV06]. Since counting subformulas impose bounds on the number of certain nodes, it may be required to duplicate $\phi$-nodes. Counters are then introduced in the Fischer-Ladner construction in order to distinguish potentially identical nodes. We now show that counters are introduced in a consistent manner. 
Lemma 4. Given a satisfying tree $T$ of a formula $\phi$, there is a tree entailing $\phi$, such that for every path from its root to a leave, there are not identical $\phi$-nodes.

Proof. If every path in $X^{T}$ does not contain identical nodes, then we are done.

Consider now the case when we have two identical nodes $n_{1}$ and $n_{2}$ in a path of $X^{T}$. Without loss of generality, we assume that $n_{1}$ is above $n_{2}$. We then proceed to build a tree $X$ from $X^{T}$, such that $n_{2}$ is grafted upon $n_{1}$. That is, the path between $n_{1}$ and $n_{2}$ is removed, not including $n_{1}$ but including $n_{2} . n_{1}$ is then linked to the subtrees of $n_{2}$. $X$ can then be seen as the pruned version of $X^{T}$.

We now show that $X$ also entails $\phi$ by induction on the derivation of $X \vdash \phi$. Most cases are immediate by the construction of $X$ and by induction.

Consider now the case of counting subformulas. Since these subformulas are true in every node, then the only important thing is to be sure that the counted nodes are not part of the pruned path. This is not possible since the counters in $n_{2}$ are the same than the ones in $n_{1}$, that is, the counters are not increased between $n_{1}$ and $n_{2}$.

Theorem 8.11 (Completeness). If a formula $\phi$ is satisfiable, then the algorithm returns 1.

Proof. By assumption, there is a (Kripke) tree $T$ satisfying $\phi$. By Lemma 4, we know there is a Fischer-Ladner tree $X^{T}$, obtained from $T$, entailing $\phi$, and whose nodes are all in $N^{\phi}$. In order to show that $X^{T}$ is produced by the algorithm, we now proceed by induction on the height of $X^{T}$.

The base case is immediate.

For the induction step, we know that the right and left subtrees of $X^{T}$, say $X_{\downarrow}$ and $X_{\rightarrow}$, are already produced by the algorithm, that is, $X_{\downarrow}, X_{\rightarrow} \in \mathcal{X}$. In order to show that $\operatorname{Update}(\mathcal{X}, Y)=\left(\mathcal{X}^{\prime}, Y^{\prime}\right)$, such that $X^{T} \in \mathcal{X}^{\prime}$, please note that $\Delta_{\downarrow}\left(n, X_{\downarrow}\right)$ and $\Delta\left(n, X_{\rightarrow}\right)$, where $n$ is the root of $X$. The fact that $n \in Y$ comes from the consistency of $\max K$ with respect to satisfaction of $\phi$, which is easily proved by an immediate induction on the structure of $\phi$.

As in [BGLS11, CGLV10, GLS07, the time complexity of the satisfiability algorithm is single exponential on the number of nodes (automaton states) introduced by the FischerLadner construction.

Theorem 8.12 (Complexity). $\mu$ TLIN satisfiability is EXPTIME-complete.

Proof. By Lemma 2, the size of the lean is at most polynomial with respect to the formula size. We then show that the complexity of the algorithm is at most exponential with respect to the lean size.

First notice that the size of $N^{\phi}$ is exponentially bounded by the lean size. Then, in the loop there is at most an exponential number of steps.

Computing the set Leaves takes exponential time since $N^{\phi}$ is traversed once.

Now note that testing the relation $\vdash$ costs linear time with respect to the size of the node. Then the entailments $\Vdash$ and $\Vdash$ take at the most exponential time .

The Update function costs at the most exponential time by the following facts: traversals on $\mathcal{X}$ and $Y$ take exponential time; and the costs of the relations $\Delta$ and \# are linear. Since each step in the loop takes at the most exponential time, we conclude that the overall complexity is single exponential.

Finally, since $\mu$ TLIN can encode all finite tree automata and is closed under negation, satisfiability is hard for EXPTIME, and hence complete. 
Recall that regular path queries (XPath) and regular tree expressions (XML schemas), extended with counting contructs, can be encoded in terms of the logical formulas with linear size with respect to the original queries and types (Theorems 3.8 and 4.5). We can then conclude that the logic can be used as an optimal query reasoning framework for XML trees.

Corollary 8.13. The emptiness, containment and equivalence of CPath queries and CTypes are decidable in EXPTIME.

\section{Conclusions}

We introduced a modal tree logic with counting and multi-directional navigation. We also showed that the logic can linearly characterize counting extensions of regular path queries (XPath) and regular tree types (XML schemas). The logic was also shown to be satisfiable in single exponential time even if the numerical constraints are coded in binary. In consequence, the logic serves as reasoning framework for XML queries and schemas extended with counting constructs. These constructs restrict the number of multi-directional regular paths. Since the logic is closed under negation, we can then decide in EXPTIME typical reasoning problems such as emptiness, containment, and equivalence of XML queries and schemas. We are currently working on the implementation of the satisfiability algorithm described in the present work with the use of Binary Decision Diagrams (BDD's), as previously described in GLS07, TTH08.

Proving correctness of programs is a crucial part in the verification of software, such as operating or real-time systems. The implementation of efficient high level program structures are often based on balanced tree structures, such as AVL trees, red-black trees, splay trees, etc. Reasoning frameworks with in-depth counting constraints, such as the ones described in this work, play a major role in the verification of balanced tree structures, as already described in Habermehl et al. [HIV10] and Manna et al. [MSZ07]. Therefore, we believe it is possible to study the field of applications of the reasoning frameworks developed in this work in the context of the verification of balanced tree structures. Also in the formal verification side, the behavior of reactive systems has been extensively studied by means of the model checking problem for the $\mu$-calculus [FM07, CGLV10]. We also consider the model checking problem for $\mu$ TLIN as as a further research direction.

Acknowledgments. This work benefited from the support of Pierre Genevès, Nabil Layaïda, Denis Lugiez and Alan Schmitt.

\section{REFERENCES}

[Bar11] Everardo Barcenas. Raisonnement automatisé sur les arbres avec des contraintes de cardinalité. PhD thesis, University of Grenoble, 2011.

$\left[\mathrm{BCG}^{+} 10\right]$ Luis Barguñó, Carles Creus, Guillem Godoy, Florent Jacquemard, and Camille Vacher. The emptiness problem for tree automata with global constraints. In LICS, pages 263-272. IEEE Computer Society, 2010.

$\left[\mathrm{BCG}^{+} 13\right]$ Luis Barguñó, Carles Creus, Guillem Godoy, Florent Jacquemard, and Camille Vacher. Decidable classes of tree automata mixing local and global constraints modulo flat theories. Logical Methods in Computer Science, 9(2), 2013. 
[BGLS11] Everardo Bárcenas, Pierre Genevès, Nabil Layaïda, and Alan Schmitt. Query reasoning on trees with types, interleaving, and counting. In Toby Walsh, editor, IJCAI, pages 718-723. IJCAI/AAAI, 2011.

[BLMV06] Piero A. Bonatti, Carsten Lutz, Aniello Murano, and Moshe Y. Vardi. The complexity of enriched mu-calculi. In Michele Bugliesi, Bart Preneel, Vladimiro Sassone, and Ingo Wegener, editors, ICALP, volume 4052 of Lecture Notes in Computer Science, pages 540-551. Springer, 2006.

[BMM09] Alessandro Bianco, Fabio Mogavero, and Aniello Murano. Graded computation tree logic. In LICS, pages 342-351. IEEE Computer Society, 2009.

[BMM10] Alessandro Bianco, Fabio Mogavero, and Aniello Murano. Graded computation tree logic with binary coding. In Anuj Dawar and Helmut Veith, editors, CSL, volume 6247 of Lecture Notes in Computer Science, pages 125-139. Springer, 2010.

[BMM12] Alessandro Bianco, Fabio Mogavero, and Aniello Murano. Graded computation tree logic. $A C M$ Trans. Comput. Log., 13(3):25, 2012.

[CD99] James Clark and Steven J. DeRose. XML path language (XPath) version 1.0. http://www.w3.org/TR/xpath.html, 1999.

[CGLV10] Diego Calvanese, Giuseppe De Giacomo, Maurizio Lenzerini, and Moshe Y. Vardi. Node selection query languages for trees. In Maria Fox and David Poole, editors, AAAI. AAAI Press, 2010.

[DL10] Stéphane Demri and Denis Lugiez. Complexity of modal logics with Presburger constraints. $J$. Applied Logic, 8(3):233-252, 2010.

[DZLM04] Silvano Dal-Zilio, Denis Lugiez, and Charles Meyssonnier. A logic you can count on. In Neil D. Jones and Xavier Leroy, editors, POPL, pages 135-146. ACM, 2004.

[FM07] Alessandro Ferrante and Aniello Murano. Enriched mu-calculi module checking. In Helmut Seidl, editor, FoSSaCS, volume 4423 of Lecture Notes in Computer Science, pages 183-197. Springer, 2007.

[Gel10] Wouter Gelade. Succinctness of regular expressions with interleaving, intersection and counting. Theor. Comput. Sci., 411(31-33):2987-2998, 2010.

[GLS07] Pierre Genevès, Nabil Layaïda, and Alan Schmitt. Efficient static analysis of XML paths and types. In Jeanne Ferrante and Kathryn S. McKinley, editors, PLDI, pages 342-351. ACM, 2007.

[HIV10] Peter Habermehl, Radu Iosif, and Tomás Vojnar. Automata-based verification of programs with tree updates. Acta Inf., 47(1):1-31, 2010.

[Hum88] Andrew Hume. A tale of two greps. Softw., Pract. Exper., 18(11):1063-1072, 1988.

[HVP05] Haruo Hosoya, Jerome Vouillon, and Benjamin C. Pierce. Regular expression types for XML. ACM Trans. Program. Lang. Syst., 27(1):46-90, 2005.

[JW96] David Janin and Igor Walukiewicz. On the expressive completeness of the propositional mucalculus with respect to monadic second order logic. In Ugo Montanari and Vladimiro Sassone, editors, CONCUR, volume 1119 of Lecture Notes in Computer Science, pages 263-277. Springer, 1996.

[KSV02] Orna Kupferman, Ulrike Sattler, and Moshe Y. Vardi. The complexity of the graded $\mu$-calculus. In Andrei Voronkov, editor, CADE, volume 2392 of Lecture Notes in Computer Science, pages 423-437. Springer, 2002.

[Mar05] Maarten Marx. Conditional XPath. ACM Trans. Database Syst., 30(4):929-959, 2005.

[MLMK05] Makoto Murata, Dongwon Lee, Murali Mani, and Kohsuke Kawaguchi. Taxonomy of XML schema languages using formal language theory. ACM Trans. Internet Techn., 5(4):660-704, 2005.

[MSZ07] Zohar Manna, Henny B. Sipma, and Ting Zhang. Verifying balanced trees. In Sergei N. Artëmov and Anil Nerode, editors, LFCS, volume 4514 of Lecture Notes in Computer Science, pages 363378. Springer, 2007.

[SSM03] Helmut Seidl, Thomas Schwentick, and Anca Muscholl. Numerical document queries. In Frank Neven, Catriel Beeri, and Tova Milo, editors, PODS, pages 155-166. ACM, 2003.

[SSMH04] Helmut Seidl, Thomas Schwentick, Anca Muscholl, and Peter Habermehl. Counting in trees for free. In Josep Díaz, Juhani Karhumäki, Arto Lepistö, and Donald Sannella, editors, ICALP, volume 3142 of Lecture Notes in Computer Science, pages 1136-1149. Springer, 2004.

[Tar55] Alfred Tarski. A lattice-theoretical fixpoint theorem and its applications. Pacific Journal of Mathematics, 5:285-309, 1955. 
[tCM09] Balder ten Cate and Maarten Marx. Axiomatizing the logical core of XPath 2.0. Theory Comput. Syst., 44(4):561-589, 2009.

[TTH08] Yoshinori Tanabe, Koichi Takahashi, and Masami Hagiya. A decision procedure for alternationfree modal mu-calculi. In Carlos Areces and Robert Goldblatt, editors, Advances in Modal Logic, pages 341-362. College Publications, 2008.

[Var98] Moshe Y. Vardi. Reasoning about the past with two-way automata. In Kim Guldstrand Larsen, Sven Skyum, and Glynn Winskel, editors, ICALP, volume 1443 of Lecture Notes in Computer Science, pages 628-641. Springer, 1998.

[WCO00] Larry Wall, Tom Christiansen, and Jon Orwant. Programming Perl - there's more than one way to do it (3. ed.). O'Reilly, 2000. 